\title{
Development and commissioning of the T6 Stalker Tunnel
}

\author{
Peter Collen ${ }^{1}$ (1) - Luke J. Doherty ${ }^{1} \cdot$ Suria D. Subiah ${ }^{1} \cdot$ Tamara Sopek $^{1} \cdot$ Ingo Jahn $^{2} \cdot$ David Gildfind $^{2}$. \\ Rowland Penty Geraets ${ }^{1} \cdot$ Rowan Gollan $^{2} \cdot$ Christopher Hambidge $^{1} \cdot$ Richard Morgan $^{2} \cdot$ Matthew McGilvray $^{1}$
}

Received: 31 December 2020 / Revised: 7 July 2021 / Accepted: 19 August 2021 / Published online: 10 October 2021

(c) The Author(s) 2021

\begin{abstract}
The T6 Stalker Tunnel is a multi-mode, high-enthalpy, transient ground test facility. It is the first of its type in the UK. The facility combines the original free-piston driver from the T3 Shock Tunnel with modified barrels from the Oxford Gun Tunnel. Depending on test requirements, it can operate as a shock tube, reflected shock tunnel or expansion tube. Commissioning tests of the free-piston driver are discussed, including the development of four baseline driver conditions using piston masses of either $36 \mathrm{~kg}$ or $89 \mathrm{~kg}$. Experimental data are presented for each operating mode, with comparison made to numerical simulations. In general, high-quality test flows are observed. The calculated enthalpy range of the experimental conditions achieved varies from $2.7 \mathrm{MJ} \mathrm{kg}^{-1}$ to $115.0 \mathrm{MJ} \mathrm{kg}^{-1}$.
\end{abstract}

\section{Graphical abstract}

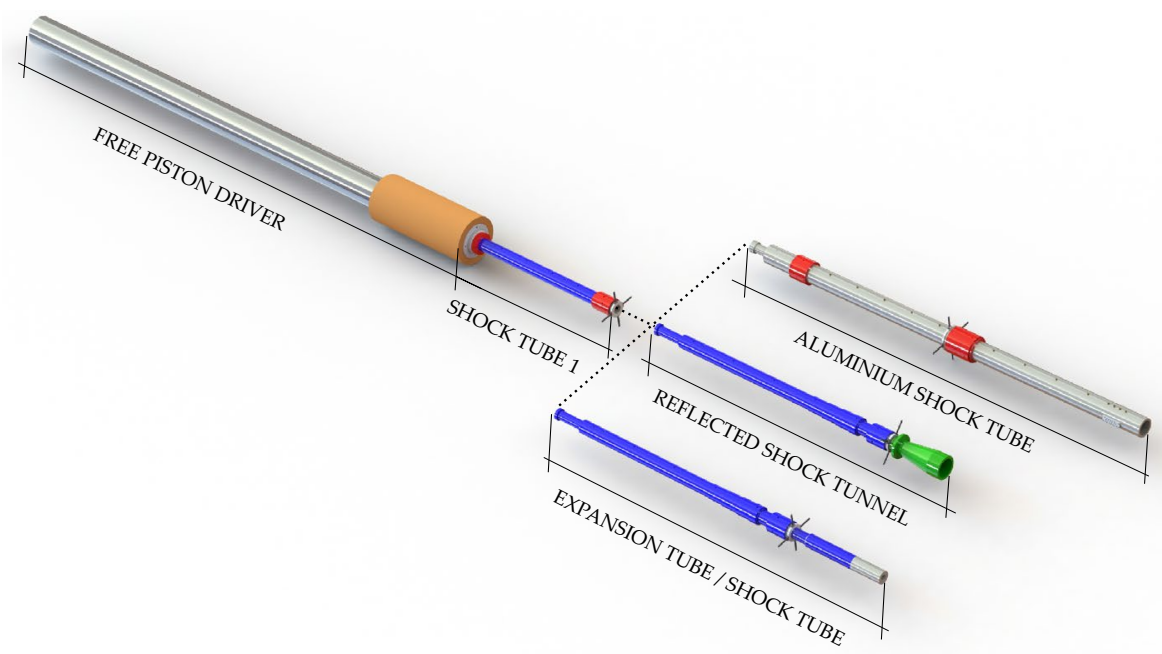

\section{Nomenclature}

A Diaphragm open area, $\mathrm{m}^{2}$

$\mathrm{f}_{\mathrm{f}} \quad$ Piston friction factor, -

$\mathrm{H}$ Total specific enthalpy, $\mathrm{MJ} \mathrm{kg}^{-1}$

$\mathrm{h} \quad$ Static specific enthalpy, $\mathrm{MJ} \mathrm{kg}^{-1}$

Peter Collen

peter.collen@eng.ox.ac.uk

1 Oxford Thermofluids Institute, University of Oxford, Southwell Building, Oxford OX2 0ES, United Kingdom

2 Centre for Hypersonics, University of Queensland, Brisbane, Queensland 4072, Australia $\mathrm{k} \quad$ Opening profile exponent, -

$\mathrm{K}_{\mathrm{L}} \quad$ Launcher loss coefficient, -

M Mach number, -

$\mathrm{P} \quad$ Pressure, $\mathrm{Pa}$

$\mathrm{t}$ Time, $\mathrm{s}$

$\mathrm{T}$ Temperature, $\mathrm{K}$

U Velocity, $\mathrm{km} \mathrm{s}^{-1}$

$\mathrm{X} \quad$ Mole fraction, $\%$

$\lambda$ Compression ratio, -

$\rho$ Density, $\mathrm{kg} \mathrm{m}^{-3}$

$\tau \quad$ Test time, $\mathrm{s}$ 
0 Total conditions

1 Driven gas initial conditions

4 Conditions at diaphragm rupture

5 Acceleration tube initial conditions

eq Flight-equivalent value

He Helium

p Pitot

$\mathrm{r} \quad$ Condition at rupture

Res Reservoir

s Shock

tr Translational-rotational

ve Vibrational-electronic

\section{Introduction}

Further development of next-generation high-speed aircraft technologies, such as air-breathing access-to-space vehicles and atmospheric entry craft, requires greater understanding of hypersonic flow phenomena. Whilst flight testing remains the most desirable source of experimental data, the prohibitive cost limits the availability of relevant results for the multitude of possible flight trajectories. Ground test facilities can undertake hypersonic experiments at a fraction of the cost of flight test programmes, providing valuable data to inform vehicle development, understand the aerothermodynamic environment, and improve computational models.

Figure 1 presents several archetypal flight trajectories of interest to hypersonics research. It is clear that the peak heating conditions for all of the cases shown occur where there are significant thermochemical effects in the flow. In addition, the majority of these missions pass through high altitudes at significant speeds resulting in large non-equilibrium regions around the vehicle. Such flow fields require accurate spatio-temporal reconstruction during ground testing. Highenthalpy test facilities are therefore required to properly recreate the flight environment and to enable relevant data to be obtained.

Stalker (2006) estimated that a continuous ground test facility capable of simulating re-entry conditions could require gigawatts of input power. This is evident in Fig. 1, which shows that continuous facilities are currently limited to maximum speeds of around $1 \mathrm{~km} \mathrm{~s}^{-1}$. Achieving higher enthalpy test conditions therefore requires a shift to transient facilities, albeit with a general reduction in achievable test time. Hypersonic pulse facilities of varying types are operated worldwide, by universities, space agencies and private companies (Lu and Marren 2002), and all continue to provide invaluable data for vehicle development. However, for trajectories such as those shown in Fig. 1 it is not possible to simulate the entirety of the flight path in any one type of ground test facility. This has led to the relatively recent conversion of existing ground test facilities to be multimodal; examples include HEK-X (Tanno et al. 2016) and X3R (Stennett et al. 2018). This challenge has also formed a core motivation for the development of the T6 Stalker Tunnel described herein.

This paper first gives details of the new University of Oxford tunnel-T6-in Section 2, describing the current arrangement and infrastructure of the facility. Results from the commissioning of the driver section with both $36-\mathrm{kg}$ and 89-kg pistons are then presented in Section 3. Progress on the characterisation of each tunnel mode is then reviewed in Sections 4 to 6 , with a range of experimental diagnostics discussed and comparisons to computational tools made. Data are presented which demonstrates the ability of T6 to simulate flight enthalpies in the range 2.7 to $115.0 \mathrm{MJ} \mathrm{kg}^{-1}$, corresponding to trajectory points for a Mach 7 flight condition
Fig. 1 Selected mission trajectories and approximate ground test facility capabilities (adapted from Fletcher (2004))

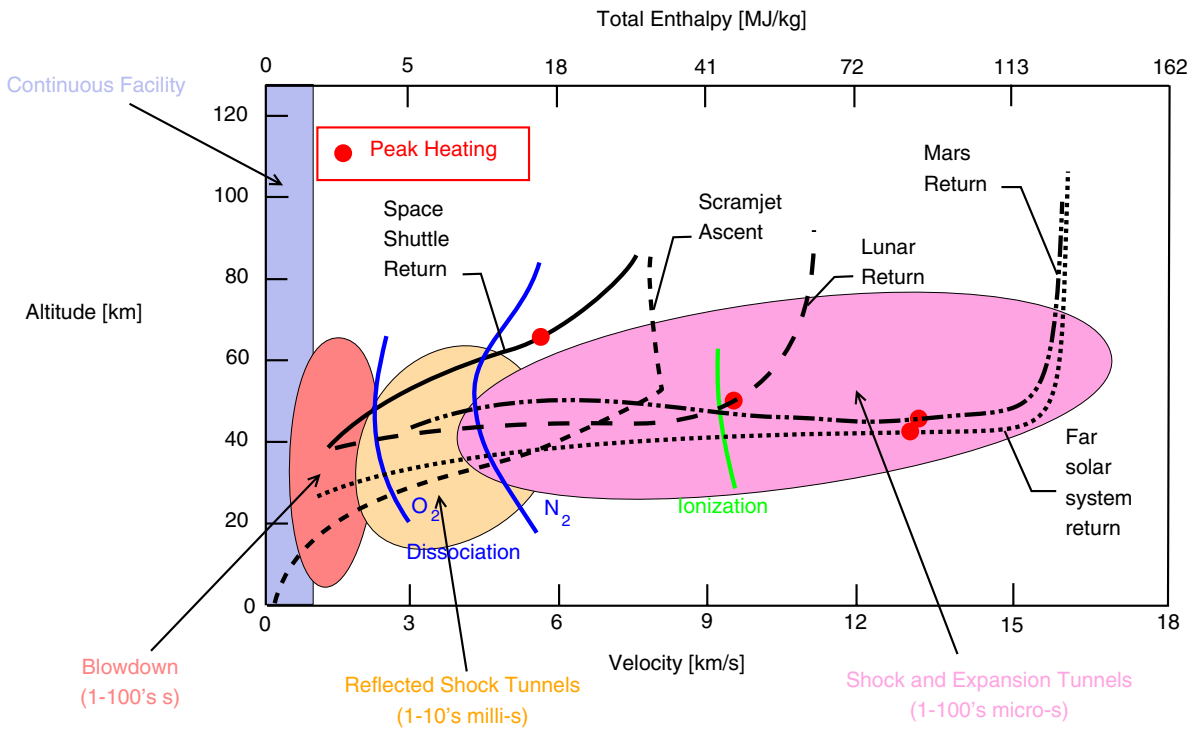


and proposed Mars Sample Return missions, respectively. Finally, Section 7 summarises the work and briefly discusses the next steps in the facility development.

\section{Facility overview}

T6 is a new multi-mode, transient, high-enthalpy aerothermodynamic ground test facility at the University of Oxford. It can operate in four distinct modes according to test requirements: Reflected Shock Tunnel (RST), Expansion Tube (ExT) and in two specialist shock tube (ST) modes. These are compared diagrammatically in Fig. 3, with the key dimensions provided in Table $1 .^{1}$ The specifics of each of these modes are discussed in subsequent sections. Also shown is the driver section, which is common to all modes. This modular nature results in a single facility capable of experimentally simulating flight conditions ranging from hypersonic atmospheric flight to hypervelocity re-entry.

\subsection{The T6 free-piston driver}

The fundamental difficulty of high-enthalpy aerothermodynamic ground testing can be summarised as follows: it is impractical or impossible to generate and contain the high stagnation pressures and temperatures required to match flight conditions through steady means. Instead, these conditions must be generated transiently. This is almost universally achieved by passing a strong shock through the test gas, increasing its stagnation properties for sufficient time to provide a useful test flow. Many variations of this process are possible, as discussed in Sect. 4-6. In all cases, a powerful driver section which is capable of driving a sufficiently strong shock through the test gas is required.

\subsubsection{T6 driver selection}

To maximise the shock speed achieved in the driven gas, the most important driver attributes are sound speed and-to a lesser extent-pressure. As a result, the operating principle of most driver technologies is to provide a source of high pressure and temperature, low molecular weight gas. The approach with the highest absolute performance is the arc driver, where the rapid discharge of electrical current through a filament greatly increases temperature and pressure in the driver section. This is the approach used by the NASA Ames EAST facility, which has recorded shock speeds in excess of $46 \mathrm{~km} \mathrm{~s}^{-1}$ (Sharma and Park 1990). However, this technology is generally not suited for applications

\footnotetext{
${ }^{1}$ Note that the reservoir is in line with the compression tube; these are labelled together in Fig. 3 as 'Free Piston Driver.'
}

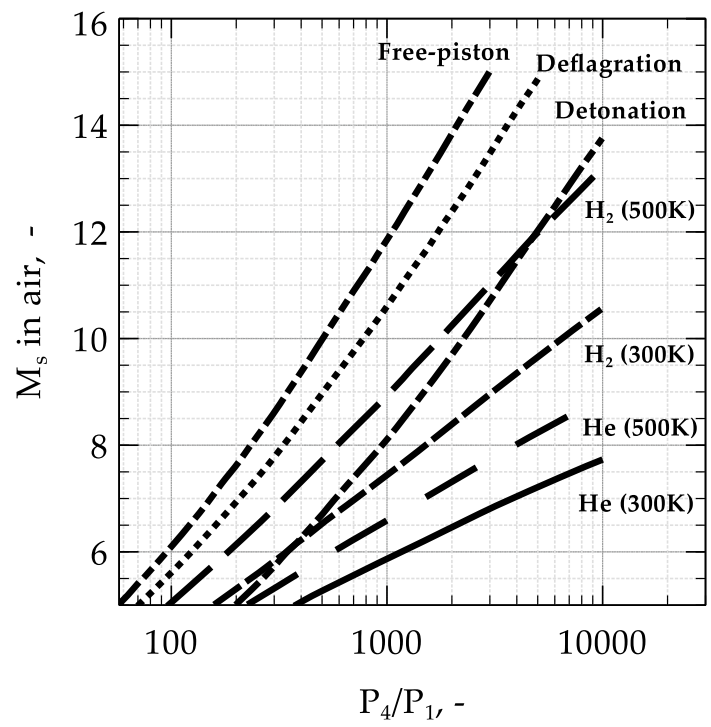

Fig. 2 Performance comparison of common driver technologies (adapted from Bakos and Erdos (1995))

which require longer test durations (i.e. model testing) due to difficulties in maintaining steady flow properties in the driver gas.

Bakos and Erdos (1995) surveyed a range of driver technologies suitable for the HYPULSE facility, considering both performance and achievable test time. Their findings are reproduced in Fig. 2, which plots the shock Mach number achievable as a function of the driver-to-driven pressure ratio. Noting the logarithmic scale of Fig. 2, cold and heated helium as well as cold hydrogen requires unfeasibly high pressures to achieve significant shock speeds. Heated hydrogen is a viable option; however, this requires very high pressures to generate high-velocity shocks. This approach is used by the CUBRC LENS tunnels, which operate at driver fill pressures up to $70 \mathrm{MPa}$ (Holden et al. 2008). This approach also has the advantage of being low in complexity. However, handling hydrogen at these pressures poses serious safety concerns which are untenable in a university setting. Similar logic can be applied to combustion (deflagration) and detonation drivers, in which a mixture of hydrogen and oxygen is either burnt or detonated to achieve the desired rupture conditions. The authors also note that it can be challenging in practice to operate a combustion driver at pressures above approximately $40 \mathrm{MPa}$ due to the occurrence of local detonations. Of the options considered in Fig. 2 the free-piston driver is shown to offer the highest performance. However, the mechanical complexity and hence capital cost of this approach are high. The knowledge required to develop driver conditions can also present significant challenges.

For the development of T6 a decommissioned but fully functional free-piston driver was fortunately available. This driver was originally part of the T3 Shock Tunnel, which 
Fig. 3 Diagram showing different $\mathrm{T} 6$ facility modes

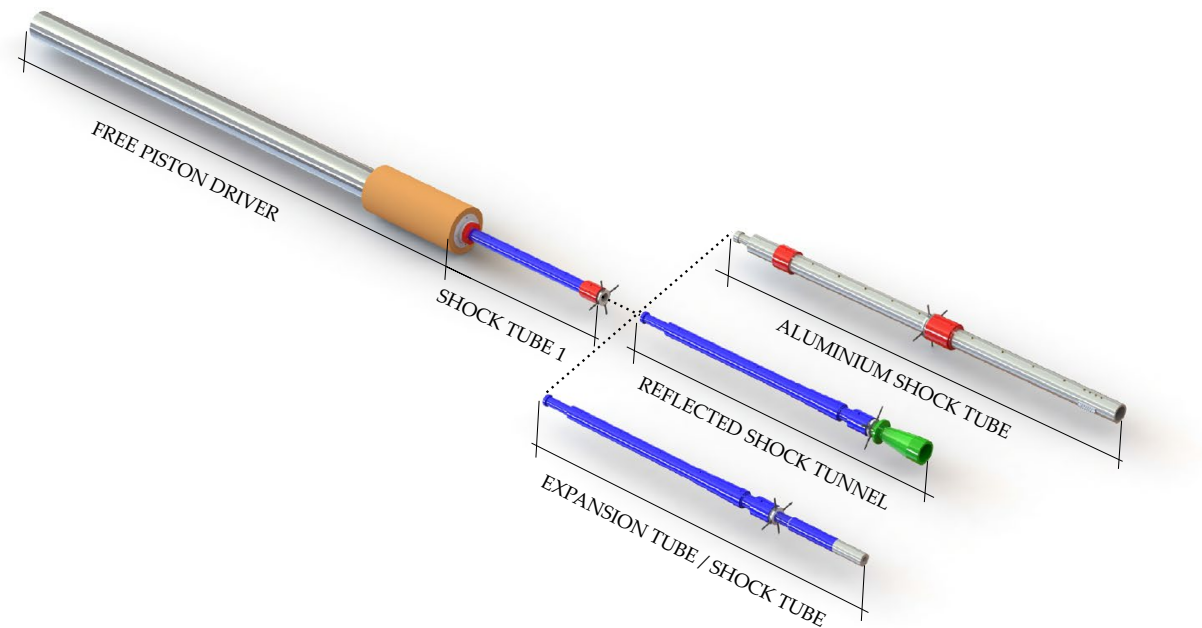

Table 1 Key dimensions of the T6 facility. Shock Tube 1 is integral to all modes; its length should thus be added to that of each mode to find the working length

\begin{tabular}{llll}
\hline Section & Length, $\mathrm{m}$ & Internal Dia., mm & Outlet Dia., mm \\
\hline Reservoir & 2.8 & 342.9 & 300.0 \\
Compression Tube & 6.0 & 300.0 & $76.2^{*}$ \\
Shock Tube 1 & 2.8 & 96.3 & 96.3 \\
$\quad$ Reflected Shock & $5.3 \dagger$ & 96.3 & $220.0 \dagger$ \\
$\quad$ Tunnel & & & \\
$\quad$ Expansion Tube/ & $6.0 \dagger$ & 96.3 & $96.3 \dagger$ \\
$\quad$ Tunnel & & & \\
$\quad$ Shock Tube & 6.0 & 96.3 & 96.3 \\
$\quad$ Aluminium Shock & 7.1 & 225.0 & 225.0 \\
$\quad$ Tube & & &
\end{tabular}

*Interchangeable orifice plate, currently matched to original T3 Shock Tube diameter.

†Variable depending on nozzle chosen. Values shown representative of this work

operated at the Australian National University from the late 1960s until the turn of the century. T3 used the first largescale free-piston driver, which proved the performance benefits and operability of the technology (Stalker 1966) and led to its adoption by several universities and national space agencies world wide (Stalker 2006). The availability of the T3 driver significantly reduced the capital cost of the freepiston design, therefore allowing the highest performance option to be chosen.

\subsubsection{T6 free-piston driver description}

In a free-piston driver, a heavy piston initially separates high-pressure reservoir gas (air) from the lower-pressure driver gas-typically a mixture of helium and argon. The tube holding this gas is termed the 'compression tube' and is bounded at the downstream end by the primary diaphragm: a metal sheet with a thickness on the order of millimetres. At the start of the test, the piston (currently either $36 \mathrm{~kg}$ or $89 \mathrm{~kg}$ ) is accelerated by the reservoir gas to velocities in excess of $150 \mathrm{~ms}^{-1}$. As the piston nears the end of the compression tube the polytropic compression of the driver gas leads to a rapid increase in pressure and temperature, consequently decelerating the piston at rates exceeding 1000 g. The increasing driver gas pressure plastically deforms the primary diaphragm until rupture. Once ruptured, the driver gas ahead of the piston expands into the low-pressure test gas in the shock tubes-driving the downstream flow processes-whilst the piston decelerates. This process is shown in the distance-time diagrams for each mode in Fig. 4.

Tanno et al. (2000) describe two requirements for piston motion at the end of the stroke: (1) the velocity should be high enough to continue compressing the driver gas after diaphragm rupture (preventing generation of expansion waves which disturb the test flow), whilst (2) decelerating to a sufficiently low speed as to avoid damage to both the piston and compression tube/primary diaphragm station components upon impact with the buffers (located at the downstream end of the compression tube). Requirements 1 and 2 are often known as 'overdriving' and 'soft landing,' respectively; driver conditions for which both are met are known as 'tuned' conditions.

The T6 driver is made up of a monolithic billet of stainless steel $9 \mathrm{~m}$ in length with an outer diameter of approximately $480 \mathrm{~mm}$. This incorporates the $6-\mathrm{m}, 300-\mathrm{mm}$ diameter honed compression tube and an in-line high-pressure air reservoir. At the primary diaphragm station, a mild steel jacket of $730 \mathrm{~mm}$ outer diameter is shrink-fitted on to the driver billet. This jacket serves a dual function, acting as an inertial mass (to limit tunnel recoil and the generation of transient stress waves in the facility) and as structural reinforcement at the primary diaphragm station. This 
Fig. 4 Distance-time diagrams of T6 shock tube modes. The nominal test time, steady expansion regions and unsteady expansion waves are denoted by $\tau$, s-x and us-X, respectively. The region of useful test gas is shaded red

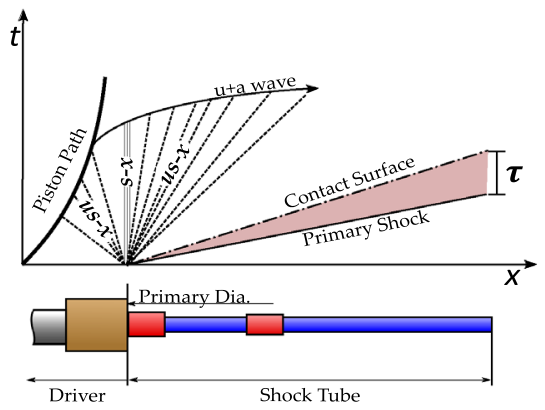

(a) Steel Shock Tube mode.

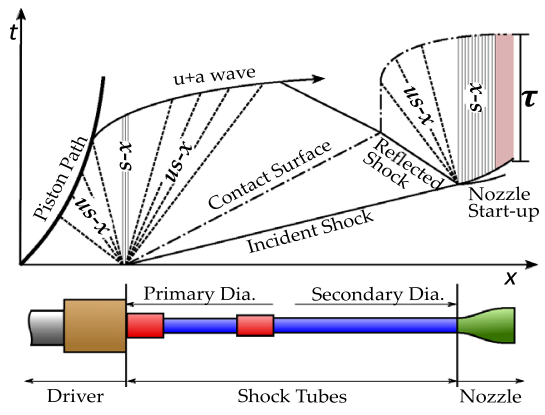

(c) Reflected Shock Tunel mode.



(b) Aluminium Shock Tube mode.

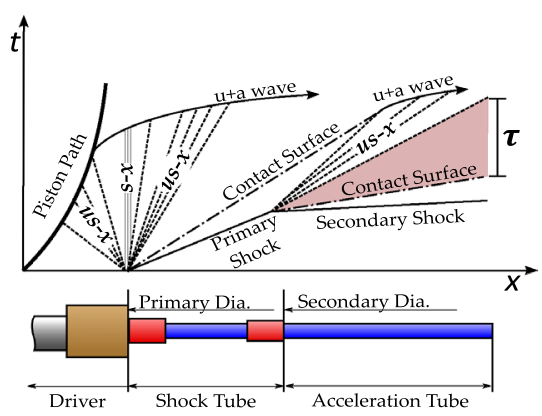

(d) Expansion Tube mode. arrangement permits a maximum diaphragm rupture pressure of $270 \mathrm{MPa}$ (Stalker 1972).

\subsection{T6 operating mode descriptions}

Figure 4 shows distance-time plots for the four operating modes of T6, starting from the moment of diaphragm rupture. These show the main wave processes which occur during a test. As shown in Fig. 3, the driver and first shock tube are common to all modes and so the left portion of each figure is identical. At the highest level, the difference between the modes is the exact mechanism through which the test flow is generated. For example, in ST mode the test gas follows directly behind the primary shock, whereas in an ExT it must subsequently undergo an unsteady expansion to the desired condition. The location of the test flow region is coloured red in Fig. 4 to aid comparison.

\subsubsection{Shock tube modes}

Shock tubes are used extensively for fundamental fluid dynamics research, chemical kinetics studies and computational code validation. This is in part due to their simplicity: they approximate one-dimensional flow phenomena, require relatively few input parameters and can-in the simplest cases-be modelled analytically. Whilst a large number of shock tubes are in use globally, the fraction active in the field of aerothermodynamics is small (Grinstead et al. 2010; Yamada et al. 2011; Brandis et al. 2010b). From the perspective of aerothermodynamic ground testing, shock tubes can be used to recreate the post-shock conditions ahead of an entry vehicle, permitting investigation of the thermochemical state of the gas. Notably, they are currently the best source of measurements of shock layer radiation, which can contribute the majority of the peak heat flux on an entry vehicle (Brandis and Johnston 2014) and cannot be accurately recreated with scaled model testing (Andrianatos et al. 2015). In general, shock tubes are not used for scale model testing; this is primarily due to the lack of an expansion process following the shock heating of the test gas. The test slug thus remains compressed, resulting in short test times. Additionally, the flow retains a high static enthalpy; the model shock layer is therefore not correctly reproduced as the Mach number is limited and hence too low for the Mach number independence principle to apply. The free stream is also highly dissociated which effects the non-equilibrium processes in the flow around the test article.

T6 has two distinct shock tube modes: the steel shock tube (SST) and aluminium shock tube (AST). The main purpose of these modes is to perform shock-layer radiation experiments, wherein a normal shock of desired velocity is passed through a quiescent gas of a pressure and composition chosen to match a trajectory point of interest. The goal of these studies is to make spatially and spectrally resolved measurements of the shock layer as it passes the test section. An analogy is then made between the evolution of the thermochemical state of the gas behind the normal shock and the stagnation line of an entry vehicle. This approach has the advantage of exactly matching the post-shock conditions to flight, with no scaling required. Depending on the 
measurement made, estimates of vehicle radiative heating, thermochemical rates and species populations can then be determined which ultimately gives a better understanding of the flight environment. Initial results from such experiments in T6 have previously been presented (Collen et al. 2019).

\subsubsection{Shock tube modes: steel shock tube mode}

The T6 SST mode consists of a steel barrel approximately 9 $\mathrm{m}$ in length with an internal diameter of $96.3 \mathrm{~mm}$. For shock layer radiation experiments, the approach as used in the X2 facility is followed, wherein the shock front is imaged as it emerges from the end of the tube (Brandis et al. 2010b). As will be shown in Sect. 4, the SST offers the better performance of the two shock tube modes-speeds in excess of $13 \mathrm{~km} \mathrm{~s}^{-1}$ have been achieved. Thus the primary purpose of the SST mode is to investigate shock layer radiation at very high entry velocities, as would be typical for vehicles on hyperbolic Earth return or Ice Giant entry trajectories. In addition, the comparatively simple configuration of the SST (i.e. no nozzle or secondary diaphragm) makes this mode ideal for the validation of computational tools and investigation of fundamental shock tube flow phenomena (Satchell et al. 2021).

Figure 4a shows a distance-time diagram of SST operation. A test condition is defined by three parameters: test gas fill pressure and composition, and the incident shock speed. The test gas initially fills the shock tube. After rupture of the primary diaphragm, the driver gas expands into the shock tube, driving a strong shock through the test gas at the desired velocity. This produces a slug of shock-processed gas which travels behind the shock, undergoing the same non-equilibrium thermochemical processes as would be observed in the stagnation line shock layer of an entry vehicle. The test time is therefore defined by the distance between the primary shock and the contact surface, which represents the boundary between the shocked test gas and expanded driver gas. The location of this test flow is shown in red in Fig. 4a.

\subsubsection{Shock tube modes: aluminium shock tube mode}

The T6 AST mode was designed specifically for shock layer radiation studies. The aluminium construction is intended to reduce the presence of carbon contaminants in emission spectra. A diaphragm at either end of the tube isolates the test gas from other sections of the facility, allowing ultimate pressures of approximately $10^{-3} \mathrm{~Pa}$ to be achieved with leak rates of $10^{-5} \mathrm{~Pa} \mathrm{~s}^{-1}$. This minimises test gas contamination due to the ingress of atmospheric air, which can significantly influence the chemical kinetics through the shock for certain compositions of interest, e.g. Titan (Brandis and Cruden 2017b). The AST features two, 200-mm-long windows at the downstream end of the tube, integrated into the tube wall. These permit optical access, with a range of window materials being available to allow transmission from the vacuum ultraviolet to the infrared (approximately $120 \mathrm{~nm}$ to $5500 \mathrm{~nm}$ ). Finally, the key attribute of the AST is its large diameter, which is increased from the nominal shock tube bore through a 12-deg half-angle conical nozzle to $225 \mathrm{~mm}$. The benefits of this are twofold: firstly, the larger diameter leads to a greater integration path length for optically thin radiation, which increases the achievable signal-to-noise ratio. Secondly, the boundary layer is relatively thinner in comparison with the tube diameter which results in a more uniform core flow, less shock deceleration and a longer slug of test gas (Mirels 1963). This extends the range of conditions at which post-shock equilibrium can be achieved.

Whilst the large diameter of the AST is favourable for radiation experiments, it results in a reduction in performance in comparison with SST mode. In the AST, the maximum shock speed achievable is reduced due to the lower pressure and temperature of the expanded driver gas downstream of the nozzle. Such a design is thus suited to the simulation of conditions where either the flight velocity or free-stream density is low, but radiative heating effects may be significant. Examples relevant to currently proposed programmes include manned Mars missions, Titan entry or high-altitude aerobraking trajectories. Similar designs (in which the nominal diameter of the shock tube is increased through an expanding section) have been employed on both the NASA Ames EAST facility and the X2 expansion tube to investigate such conditions (Cruden 2012; Jacobs et al. 2015; Cruden et al. 2014; Brandis and Cruden 2017b).

The distance-time diagram for the AST mode of operation is shown in Fig. 4b. The experiment proceeds identically to those conducted in SST mode, with the exception that the test gas now initially sits in the AST. The shocked gas from the first tube-followed by the driver gas-then expands through the conical nozzle into the AST, driving the secondary shock into the test gas. As previously discussed, the expansion results in a reduction in shock speed. It is this shock that generates the test flow; this is again shown in red in Fig. 4b.

\subsubsection{Reflected shock tunnel mode}

Reflected shock tunnels have been used extensively over the past half-century to study a range of high-enthalpy aerothermodynamic problems, including scramjet engine testing, boundary layer transition and hydrogen ionisation studiese.g. Stalker et al. (2005); Stalker (2006). Physically, the difference between RST and SST modes is the presence of a converging-diverging nozzle at the downstream end of the facility, as shown in Fig. 3. Currently, T6 has both Mach 7 and 8 nozzles available. Unlike the ST modes, RST mode is 
primarily meant for the testing of scaled models. The nozzle expansion provides a significant core flow region; models may therefore be made a sufficient size to permit high-resolution measurements of surface properties (e.g. pressure, heat flux) and aerodynamic forces. As RSTs typically have test times between $1 \mathrm{~ms}$ and $10 \mathrm{~ms}$, they are particularly suitable for the investigation of relatively long time-scale phenomena where flow enthalpy is important, such as combustion. Many large-scale RST facilities operate world wide, with capability to test up to orbital conditions (Hannemann et al. 2016; Stennett et al. 2020; Itoh et al. 1998; Mundt 2016; Wang et al. 2017; Tsai et al. 2009).

Figure $4 \mathrm{c}$ shows an idealised distance-time diagram of T6 in RST mode. Initially, the test gas sits in the shock tube between the primary diaphragm and a secondary, Mylar diaphragm near the nozzle throat. As in SST mode, the test gas is first processed by the primary shock. This shock then reflects from the nozzle throat region, stagnating the test gas via a reflected shock process. This stagnated gas then acts as the supply reservoir for the nozzle flow, permitting aerothermodynamic testing of scaled models at matched total enthalpy and Reynolds number. The Mach number is often not matched as nozzles are typically designed for fixed Mach numbers and are affected by variations in stagnation enthalpy. This is sufficient for many hypersonic aerothermodynamic studies due to the Mach number independence principle. However, it is possible to fully match a flight condition-including Mach number-when operating a properly designed nozzle at its nominal enthalpy, e.g. Doherty et al. (2015).

The test time in a reflected shock tunnel is defined by the duration of steady conditions in the nozzle supply region. This is typically limited by three fundamental mechanisms: drainage of the available test gas and hence ingestion of the contact surface into the nozzle, the arrival of expansion waves from the decaying pressure in the driver and the motion of the contact surface after passage of the reflected shock. The former is the scenario shown in Fig. $4 \mathrm{c}$ and is defined by the geometry of the facility, in particular the shock tube length and the relative area of the nozzle throat and shock tube bore. The second effect-arrival of expansion waves from the driver (the $u+a$ wave in the plots of Fig. 4) — can be sufficiently delayed through the use of a free piston. This is achieved through the 'overdriving' technique discussed in Section 2.1. The final considerationthe motion of the contact surface-presents the greatest challenge for condition design. If the Mach number of the reflected shock in the expanded driver gas and shocked test gas is equal, the reflected shock will pass through the interface unhindered and stagnate it as shown in Fig. 4c. Conversely, a mismatch in Mach number will cause the contact surface to either accelerate downstream or to reverse its motion. This leads to a corresponding departure from the nominal steady conditions in the test gas through an arrival of either a third shock or an expansion fan, respectively. The achievement of a stationary contact surface is known as tailoring (Wittliff 1959). In reality, this process is more complex as the contact surface is an extended region of vortical structures, the shape of which is highly dependent on the upstream flow geometry and diaphragm opening processes (Goozée et al. 2006). In addition, the presence of a boundary layer can cause complex interactions when the reflected shock meets the contact surface, including jetting of driver gas near the wall (Stalker and Crane 1978) which results in contamination of the test flow.

\subsubsection{Expansion tube mode}

As flight total pressure and enthalpy increase, it becomes increasingly difficult to reproduce test conditions of interest in a ground test facility which employs a nozzle stagnation region. From a practical perspective, the high-temperature and pressure test gas must be safely contained for the duration of the test. Erosion of the nozzle throat has been observed in several large reflected shock tunnel facilities, increasing costs of test campaigns and resulting in uncertainty in flow conditions. In addition, reflected shock tunnels also exhibit issues with residual free-stream non-equilibrium thermochemistry as stagnation enthalpy is increased (Hornung 1990; MacLean and Holden 2006). Expansion tubes, first proposed by Trimpi (1962), offer the potential to reach significantly higher velocities, with total pressures on the order of gigapascals, whilst significantly reducing the level of thermochemical non-equilibrium in the free stream. This is achieved by increasing the total enthalpy of the test flow through an unsteady expansion process which avoids stagnating the gas. These advantages come at the expense of test time, which is typically on the order of $10-100 \mu$ s. Expansion tubes are of particular use for conditions with high total pressures, such as low-altitude, high Mach number scramjet experiments and atmospheric entry (Gildfind et al. 2016). In general, expansion tubes are used for scaled model testing. Although less common than RSTs, several researchlevel expansion tubes are in active use (Gildfind et al. 2016; Tanno et al. 2016; Tamagno et al. 1990; Dufrene et al. 2007; MacLean et al. 2013).

Figure $4 \mathrm{~d}$ presents a distance-time diagram showing the theoretical operation of T6 in expansion tube mode. As in SST mode, the test gas is initially processed by an incident shock generated by rupture of the primary diaphragm. A thin secondary diaphragm (either aluminium foil or Mylar) at the end of the shock tube initially separates this test gas from a region of very low-pressure laboratory air, known as the accelerator gas. When the primary shock reaches this diaphragm the diaphragm ruptures, allowing the highpressure, shock-heated test gas to unsteadily expand into 
Fig. 5 Components used for T6 free-piston driver blank-off tests

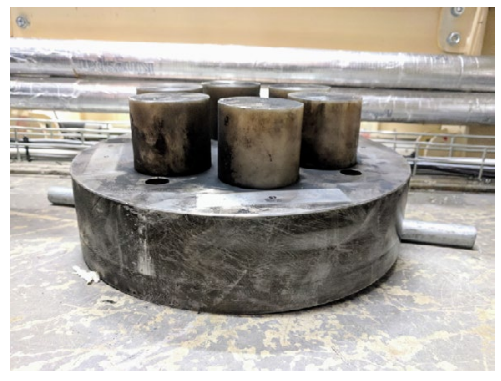

(a) Photograph of blanking plate with nylon buffers, taken after testing.

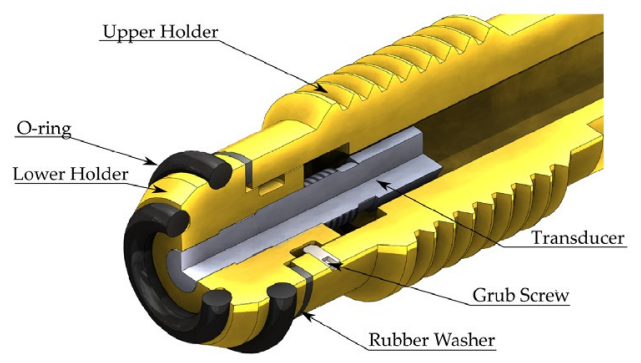

(b) Detail view of blanking plate transducer mount. the acceleration tube. This supersonic unsteady expansion process results in a net increase in total enthalpy, as given by Eq. (1) (from Trimpi (1962)). The region of shocked and expanded shock tube gas makes up the test flow, as shown in Fig. 4d. At high-velocity conditions, the test time is typically limited by the arrival of the unsteady expansion fan (which follows the test gas) at the test model.

$d H=-(M-1) d h$

\section{Free-piston driver commissioning}

A prerequisite for achieving high performance and safe operation of a free-piston driver is a thorough understanding of the piston dynamics. The multi-modal nature of T6 necessitates a range of driver conditions to enable testing across the wide range of desired flight trajectories. This section details the development and commissioning of four driver conditions using two different piston masses of $36 \mathrm{~kg}$ and $89 \mathrm{~kg}$. Similar conditions were previously used in T3 and produced total enthalpies from $3.0 \mathrm{MJ} \mathrm{kg}^{-1}$ to $39.0 \mathrm{MJ} \mathrm{kg}^{-1}$. This range is sufficient to meet current test requirements; however, further driver conditions will be developed as necessary to support future test campaigns.

To characterise the motion of the piston, commissioning tests are performed with a thick blanking plate (designed to prevent rupture) in place of the diaphragm. This 'blank-off test' arrangement provides the necessary experimental data to calibrate numerical predictions of the piston motion. The approach is as follows: the greatest risk to the facility occurs after diaphragm rupture due to the loss of cushioning driver gas from in front of the piston. In a blank-off test, no rupture occurs and the piston is thus prevented from striking the buffers. The blanking plate contains a pressure transducer, which allows inference of the piston motion and is the primary measurement used to calibrate a computational model of the free-piston driver (Section 3.2). This can then be used to predict the piston impact speed during a real test. The plate must incorporate pressure instrumentation as, unlike more modern free-piston tunnels, the T6 driver does not feature a pressure transducer at the primary diaphragm station.

\subsection{Driver commissioning: experimental arrangement}

The blanking plate used in lieu of the primary diaphragm is shown in Fig. 5a and was manufactured from S355 mild steel with a total thickness of $70 \mathrm{~mm}$. The blanking plate was fitted with six nylon buffers, $60 \mathrm{~mm}$ in diameter and $55 \mathrm{~mm}$ in length to further reduce the risk of damage. The thickness of the plate allows for a design pressure of 100 $\mathrm{MPa}$ and permits inclusion of the pressure transducer. Previous studies employing piezoelectric pressure transducers in shock tunnels have experienced issues with excessive noise, which is typically attributed to transmission of highfrequency vibrations through the facility structure (Gildfind et al. 2013; Wagner 2018). Based on these observations, attempts were made in the current work to ensure the pressure transducer was vibrationally isolated from the facility.

A section view of the resulting transducer holder assembly is given in Fig. 5. Made of brass, this transducer mount was designed such that there was no path of continuous metal-on-metal contact between the sensor and the facility. The transducer (a PCB Piezotronics series CA102B03, removed from the factory-supplied threaded mounting adapter) is screwed into the lower half of the assembly, which is separated from the blanking plate by two nitrile o-rings. The upper half is isolated from the lower half by a rubber washer. The rubber washer offers some degree of vibration isolation whilst also allowing sufficient contact to retain the sensor against the high pressures experienced at the end of the compression tube. Two grub screws allow retrieval of the transducer and lower holder half sub-assembly. A circumferential groove ensures that these grub screws are not in contact with the lower half when the assembly is fully tightened.

The original 36-kg and 90-kg T3 pistons were refurbished for the testing reported here. The refurbishment consisted of re-skimming the outer diameter of the piston body, replacing the downstream seal and wear rings and changing their 
Table 2 Developed T6 Driver Conditions

\begin{tabular}{llllll}
\hline Piston & Condition & $\mathrm{P}_{\mathrm{Res}}, \mathrm{kPa}$ & $\mathrm{P}_{4}, \mathrm{MPa}$ & $\mathrm{X}_{\mathrm{He}}, \%$ & $\lambda_{\mathrm{r}},-$ \\
\hline Aluminium (36 kg) & $\mathrm{A}$ & 730 & 9.8 & 0 & 60 \\
& $\mathrm{~B}$ & 2100 & 29.6 & 100 & 60 \\
& $\mathrm{C}$ & 3500 & 46.2 & 100 & 60 \\
St. Steel (89 kg) & HA & 5500 & 20.6 & 19 & 22 \\
\hline
\end{tabular}

Table 3 L1d3 model tuning parameters to match blank-off data

\begin{tabular}{lll}
\hline Piston & $\mathrm{K}_{\mathrm{L}}$ & $\mathrm{f}_{\mathrm{f}}$ \\
\hline Aluminium $(36 \mathrm{~kg})$ & 3.4 & 0.275 \\
St. Steel $(89 \mathrm{~kg})$ & 3.7 & 0.880 \\
\hline
\end{tabular}

material to oil-impregnated nylon. A screw thread was also added to the front face of each piston to facilitate retrieval from the compression tube. These modifications have resulted in a reduction in piston mass to $35.8 \mathrm{~kg}$ and 88.6 $\mathrm{kg}$. Approximately 20 blank-off tests were conducted, leading to the development of four driver conditions. These are summarised in Table 2, where each condition is defined in terms of its helium mole fraction (with the balance made up by argon), nominal rupture pressure and compression ratio at rupture. For completeness, the required reservoir pressure is also included.

\subsection{Driver commissioning: results}

The purpose of blank-off testing is to tune computational models, building confidence in the predictions of piston trajectory. The numerical code chosen in this work to model the facility was L1d3, a quasi-one-dimensional transient Lagrangian code with equilibrium chemistry capability (Jacobs 1998). This code has been shown to accurately reproduce experimental results in many other free-piston facilities at similar conditions (Mundt et al. 2007).

Translation of the three-dimensional geometry of a real facility into the one-dimensional code causes an inherent loss of fidelity. It has been shown that better agreement can be achieved by ensuring that the volume of each section of facility is reproduced accurately in the computational geometry (Andrianatos et al. 2016). However, fully twoand three-dimensional flow features can never be captured by L1d3 and so loss factors are used to account for their effect. The two sources of loss considered are the minor pressure loss coefficient $\left(\mathrm{K}_{\mathrm{L}}\right)$ through the piston launcher (where the flow is forced to expand radially) and the piston seal friction factor $\left(\mathrm{f}_{\mathrm{f}}\right)$. Table 3 presents the values of these tuning parameters which gave the best match to the experimental data for each piston. In the case of the aluminium piston, where multiple conditions have been tested, a single set of loss factors was found to match well across all conditions. These values also compare favourably with those found for the X2 expansion tube by Gildfind (2012), which has a similar launcher geometry. The results for the stainless steel piston launcher loss coefficient also show good agreement with these values. The piston friction factor is significantly higher for the stainless steel piston than that found for the aluminium piston. This difference is attributed to a tighter fit of the stainless steel piston's nylon wear rings in the compression tube bore due to a revision of manufacturing tolerances between the refurbishment of the two pistons.

Representative experimental pressure traces are shown in Fig. 6 alongside the matched L1d3 results for the four conditions tested. In each case the pressure traces have been time-referenced to align the peak values of the numerical and experimental results. The secondary waves which occur when argon is used as the driver gas complicate manual identification of the peak pressure times. As a result, the peak was identified by fitting a curve (arbitrarily chosen as a fourth-order Gaussian) to the experimental and computational data for each condition. Whilst there is no specific physical basis for this choice of fitting function, it was found to give good agreement for the computational results for which the exact point of piston reversal is known. This approach was thus deemed sufficient to also fit the experimental traces.

The results of Fig. 6 demonstrate good overall agreement between the computational model and experiments, as seen both in the peak pressure values and in the timing of the argon secondary wave processes visible in Figs. $6 \mathrm{a}$ and d. The stronger damping of these secondary waves in the experimental data is due to the pressure transducer being mounted in a recessed cavity which results in a longer rise time. This was not modelled in L1d3. The initial pressure rise is well matched; however, the computational model slightly overpredicts the pressure during piston rebound. This effect is likely due to heat loss from the driver gas; L1d3 uses a pipe flow model to calculate heat loss which performs poorly at the low velocities experienced during blank-off tests (Gildfind 2012). In addition, during the pressure peak the sensor is exposed to high gas temperatures (in excess of $4500 \mathrm{~K}$ ). This causes thermal strains to be generated in the piezoelectric transducer which can result in an apparent fall in pressure. Whilst this was mitigated by coating the front of the transducer with silicone grease, the extent to which this effect is still present is not easily quantified.

The close agreement with the experimental results suggests that the L1d3 model can be trusted to accurately predict piston motion. These tuned parameters have since been used to model facility performance for the experiments presented in the subsequent sections. 
Fig. 6 Representative results from free-piston blank-off tests with comparison to matched L1d3 model
Fig. 7 Performance predictions of T6 ST modes with air test gas, alongside Earth entry trajectories (adapted from McGilvray et al. (2015)) with tested conditions shown

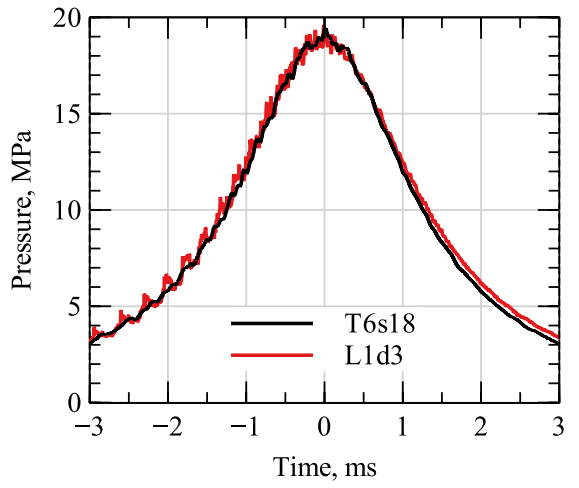

(a) Condition A.

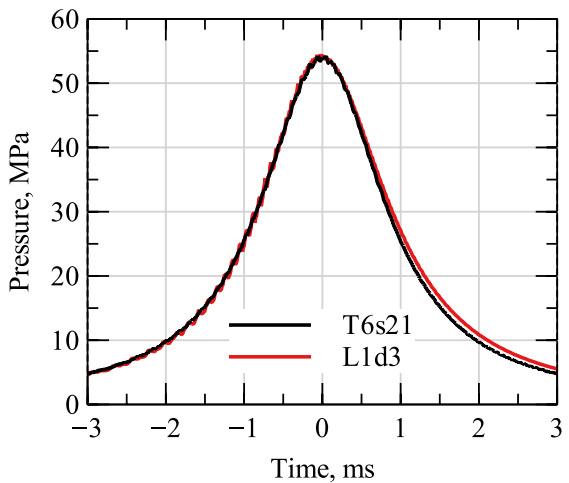

(c) Condition C.

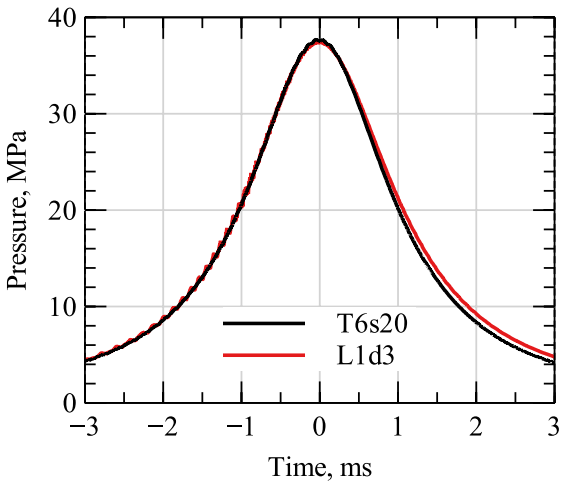

(b) Condition B.

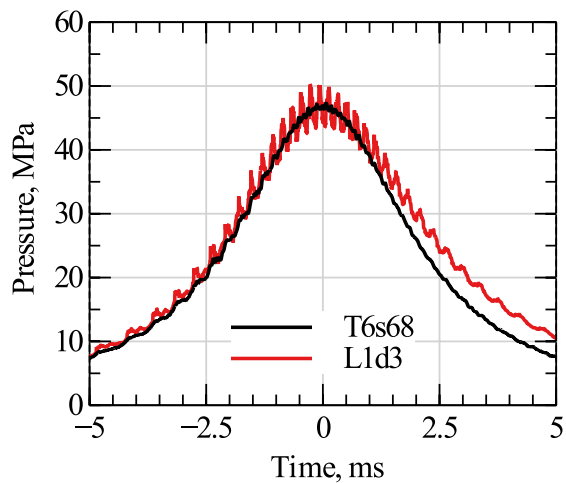

(d) Condition HA.

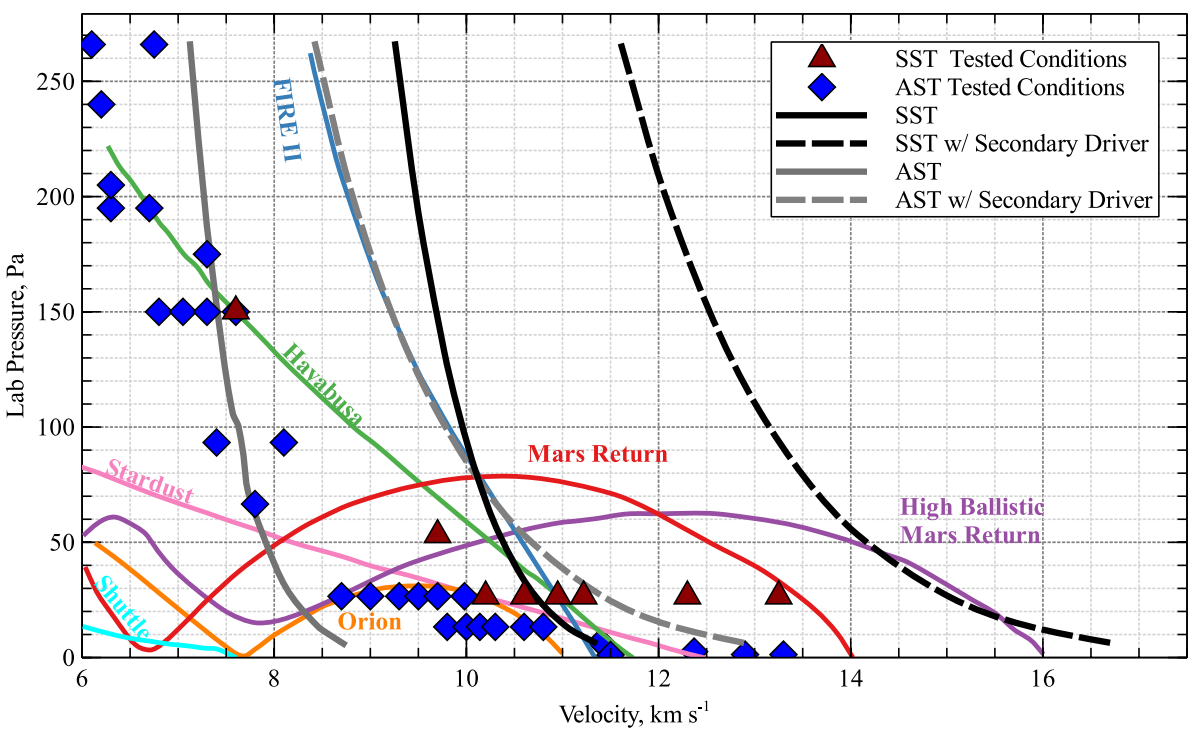

\section{Shock tube modes}

Figure 7 shows theoretical performance predictions of both T6 shock tube modes at conditions of interest for shock-layer radiation studies calculated using PITOT: a state-to-state equilibrium analytic code for high enthalpy pulse facilities (James et al. 2018). This analysis is based on driver condition $\mathrm{C}$, i.e. that with the highest performance, as detailed in Table 2. These predictions are overlaid on example trajectories of Earth entry missions of interest. Also shown in Fig. 7 are the test points reached to date in both modes. The vast majority of tests in SST 
mode have targeted $10 \mathrm{k} \mathrm{ms}^{-1}$ and 0.2 Torr (26.6 Pa), a benchmark condition for shock layer radiation (Brandis and Cruden 2017a). Tests at similar conditions have also been undertaken in AST mode, where significant portions of the Orion return trajectory and low-speed Hayabusa entry conditions have been investigated. These are also identified in Fig. 7.

Figure 7 also shows the theoretical performance achievable through addition of a secondary driver. The secondary driver is an intermediate tube (for T6, Shock Tube 1 is used; see Fig. 3), typically filled with helium, between the main free-piston driver and the shock tube; this can permit faster shock speeds to be achieved in the test gas. A full analysis of the mechanisms by which this performance augmentation is achieved is provided by Gildfind et al. (2015). Commissioning of the secondary driver section is on-going. However, predictions suggest the majority of the presented flight trajectory points could be reached in SST mode with a secondary driver, with theoretical speeds in excess of $15 \mathrm{~km} \mathrm{~s}^{-1}$ achievable. To date, the fastest shock speed achieved in combination with a secondary driver is $13.3 \mathrm{~km} \mathrm{~s}^{-1}$.

\subsection{Shock tube modes: experimental arrangement}

The shock speed variation through the facility is determined using time-of-arrival calculations between PCB Piezotronics 113 and 132 series pressure transducers flush-mounted in the shock tube wall. In the SST mode, 12 shock timing station positions are distributed along the length of the facility. In comparison, the AST mode has 17 measurement locations; four of these are clustered around the optical windows at the end of the tube to better resolve the shock speed variation at the measurement location.

The arrival time of the shock at each sensor is extracted automatically using the method of James (2019), wherein the wall pressure traces are processed using a Canny edge detection algorithm to detect the shock location. The shock speed is then calculated using the time-of-flight between the transducer locations. In this work, the values for shock speed are plotted at the central point between the positions used for the calculation.

The uncertainties in shock speed are calculated using the method detailed in Appendix 2 of James et al. (2018), which accounts for uncertainty in both the distance between transducers and the arrival time. Due to the similarity of experimental arrangement, the distance uncertainty was assumed to be identical to that of those authors with a value of \pm $2 \mathrm{~mm}$ used for each transducer location. The error in the arrival time is made up of two components: the data acquisition system sampling period and the uncertainty in the shock arrival time itself. In this work, all data were recorded with a National Instruments 6368 series card at its maximum



(a) Condition $\mathrm{A}, \mathrm{P}_{1}=1000 \mathrm{~Pa}$.

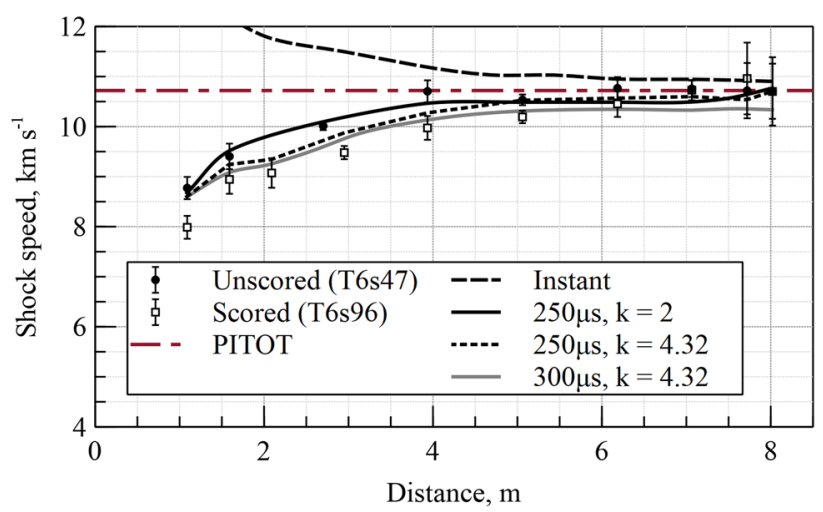

(b) Condition C, $\mathrm{P}_{1}=26.6 \mathrm{~Pa}$.

Fig. 8 Finite opening time diaphragm simulations using L1d3 (greyscale lines) with comparison to experiment (points). Also shown is the prediction of the PITOT code (dash-dot red line). All tests were conducted in laboratory air

sampling frequency of $2 \mathrm{MHz}$. The uncertainty in the shock arrival time is assigned by the experimenter by manually selecting the period in which they are certain the shock has arrived. The error is typically largest at the downstream end of the tube (where the transducers have the smallest spacing and therefore are most sensitive to uncertainties in transducer spacing and transit time) and at the beginning, where the sensors used in this work had a lower sensitivity and therefore the arrival of the shock cannot be identified with the same level of confidence.

\subsection{Shock tube modes: SST experimental results}

Representative shock speed data for driver conditions A and $\mathrm{C}$ (as detailed in Table 2) are given in Fig. 8. For the tests reported here, laboratory air (defined in this work as ambient air from the experimental area) was used as gas purity was not expected to have a significant effect on the observed performance. In general, good agreement is observed between the experimental measurements and the 
predictions of PITOT at the downstream end of the tube. For all cases an initial ramp-up in shock speed is observed, which is attributed to the finite opening time of the primary diaphragm. This effect has been noted by several authors, e.g. White (1958).

The T6 diaphragms are either unscored, where a plain sheet of material is used, or scored, in which case a mark is machined or stamped into the surface of the diaphragm. Scored diaphragms are generally preferred, as the rupture pressure can be more finely controlled via the depth of the score. Furthermore, smaller and fewer fragments of the diaphragm detach if it is scored, reducing the risk of damage to expensive models in the test section or to the tunnel itself. However, the rupture pressure of scored diaphragms is challenging to predict and can exhibit wide variation depending on the particular technician, manufacturing process, cutting tool and material used. Whilst the T6 driver pressure is not measured directly, variability in rupture conditions is observed through the shock speed achieved. Other facilities using scored diaphragms have reported differences in burst pressure of up to $30 \%$ (Boyer 1965).

The plots of Fig. 8 show shock speed variation for both unscored and scored diaphragms at both conditions. Whilst the differences between the aluminium diaphragm tests of Fig. 8a are small, the qualitative trend of shock speed between scored and unscored shows greater differences for the case of Fig. 8b. Here, the unscored test reaches an essentially steady speed by approximately 4 metres, whereas with scored diaphragms shock speed monotonically increases until almost 6 metres. The reasons for this are explored in greater detail in Section 4.3. In either case, for the majority of the tube length this variation is small in the context of other facilities which perform shock layer radiation experiments (Chandel et al. 2019).

\subsection{Shock tube modes: facility modelling}

The ability to accurately predict shock speed variation is essential for the design of pulse facility experiments. The magnitude of shock layer radiation is particularly sensitive to changes in shock speed (Brandis et al. 2010a). Shock speed variation is also critical when the facility is operated with a secondary driver or in expansion tube mode (see Section 6). In those tests, the secondary diaphragm is at a distance of approximately $3 \mathrm{~m}$ along the tube. From Fig. 8, diaphragm opening processes dominate the shock speed profile in this region and therefore the performance in these modes is strongly coupled to these opening effects. Understanding the mechanisms by which shock speed variation occurs in the test facility is thus fundamental to the design of high-performance test conditions. Furthermore, given the high capital and labour cost of tunnel test campaigns, it is desirable to develop a predictive capability for shock tube performance which can be used to investigate test conditions ex ante.

To investigate differences in shock speed variation a model of the whole facility was developed using the L1d3 code. The model used was identical to that used in Section 3.2 with the addition of the tunnel geometry downstream of the primary diaphragm. The test gas was modelled as equilibrium air, using a look-up table generated from the NASA Chemical Equilibrium with Applications code of Gordon and McBride (1994). The boundary-layer mass loss models of Doolan and Jacobs (1996) were also applied in the test gas to better capture shock deceleration due to the Mirels effect. For the shots represented in Fig. 8b their laminar correlation was used, whereas for simulations of the experiments of Fig. 8a the turbulent formulation was applied due to the significantly higher shock tube fill pressure. This distinction was made based on the guidelines presented by Mirels (1964).

Two simulation methodologies were used: first, the diaphragm was modelled with the traditional approach used in the code, in which it ruptures instantaneously at a given pressure. Secondly, to better capture the influence of finite diaphragm opening time, a variable-area boundary condition was applied in place of the diaphragm. This feature was initially developed in the thesis of Doolabh (2016) and was primarily meant for the modelling of valves in Ludwieg Tube facilities. However, it essentially allows arbitrary transient variations in area to be applied which can be used to model diaphragm opening profiles from the literature.

Two factors influence the downstream variation of shock speed during diaphragm opening: the overall duration of the opening process and the variation in area during this time. The model used for the variation in area is given in Eq. (2). Two approaches were used: the first was a parabolic curve $(\mathrm{k}=2)$ which is a reasonable approximation to the analytic calculation of Simpson et al. (1967). The second model was based on the experimental observation by Rothkopf and Low (1974) that whilst the aluminium opening behaviour closely followed the 'ideal' predictions of Simpson et al. (1967), the initial tearing of the petals of a ductile diaphragm can take up to $50 \%$ of the total opening time, with only $5 \%$ of the area being open by this point. This suggests a very different variation in area (or 'opening profile') for the same total opening time. Rearranging Eq. (2) to solve for $\mathrm{k}$ and using 0.5 and 0.05 for the normalised time and area, respectively (representing only $5 \%$ of the full area being open after $50 \%$ of the total opening time), a value can be calculated for $\mathrm{k}$ of 4.32 .

For the T6 experiments, the aluminium diaphragms were of grade $1050-\mathrm{H} 14 \mathrm{~A}$ with an elongation at failure of $10 \%$ to $15 \%$ according to the material certificate. The stainless steel diaphragms were manufactured from grade 304 2B sheet; these had an elongation at failure of $55 \%$ to $60 \%$, again determined from the provided data for the specific 
batch of material. Given the large difference in ductility for the two diaphragm materials used it was anticipated that this property could be germane to the observed opening profiles of Fig. 8. Although the findings of Rothkopf and Low (1974) regarding ductility were based on copper and brass diaphragms of unknown specification, the stainless steel used in the T6 experiments has a measured elongation equivalent to (or exceeding) common ranges for ductile alloys of these materials. In this work, we therefore assume that the scored stainless steel diaphragms can be modelled using the ductile value for $\mathrm{k}$ (i.e. 4.32).

$\frac{A}{A_{o}}=\left[\frac{t}{t_{o}}\right]^{k}$

The results of these simulations are also compared with the experimental data in Fig. 8. For both cases of Fig. 8 it can be seen that with the instantaneous opening model the initial trend in shock speed is not well captured, although there is reasonable agreement by the end of the tube. In contrast, the finite opening time results recreate the observed shock speed profile accurately along the entire tube length.

Considering the data of Fig. 8a, a parabolic opening model with an opening time of $200 \mu$ s appears to provide the best match to the experimental data. The slower opening profile also produces a similar trend in shock speed, albeit with an additional brief increase at around 2 metres. Unfortunately, this was not resolved in the experiment given the transducer spacing used. The negligible difference in shock profiles is likely due to the relatively low overall shock speed: at a nominal velocity of $3 \mathrm{~km} \mathrm{~s}^{-1}$, the shock will have travelled only 0.6 metres in the total diaphragm opening time. As a result, the largest differences in opening profile are upstream of the first pressure transducer and are therefore not observed experimentally.

The results for the L1d3 simulations at condition C are compared with the experimental data in Fig. 8b. The unscored experimental results are best matched by a parabolic opening profile with a total duration of $250 \mu$ s. Notably, the steady-state velocity is achieved in both simulation and experiment by approximately $4 \mathrm{~m}$. Conversely, the scored diaphragm results are best matched with the slower opening profile $(\mathrm{k}=4.32)$ at an opening time of $300 \mu \mathrm{s}$. This model accurately captures the gradual rise in shock speed along the tube. Also shown for comparison is the effect of solely changing the opening profile, whilst maintaining the total opening time at $250 \mu \mathrm{s}$. It can be seen that the majority of the difference in the shock speed variation in the first half of the tube stems from the opening profile used, rather than simply the overall opening time.

The scored results are consistent with the conclusions of Rothkopf and Low (1974) regarding the slow initial opening of ductile materials. However, it is not immediately clear why the scored and unscored behaviour is so different, given that the material is identical. The majority of the disparity in opening profile is attributed to tearing of the score marks by Rothkopf and Low (1974), and so it may be that the failure mechanism of the unscored diaphragms is fundamentally different. In addition, the diaphragm opening is a complex, three-dimensional process for which the presented model presents a significant simplification. Nonetheless, good agreement with experiment is observed and the current level of fidelity is judged sufficient for the initial design of facility test conditions.

The results presented here demonstrate that to properly model the shock speed variation in a high-enthalpy shock tube requires consideration of the diaphragm opening behaviour. Two parameters - the total opening time and the area variation throughout the opening-are shown to influence the evolution of shock velocity over the length of the facility. This modelling approach yields improved agreement with the experimentally measured shock speed profile. Based on the results of this study, work is currently on-going to trial less ductile primary diaphragm materials for condition C, with the objective of further increasing facility performance.

\subsection{Shock tube modes: AST experimental results}

Representative shock speed variation for driver conditions $A$ and $C$ is shown in Fig. 9. In both cases scored diaphragms were used. The behaviour up to 3 metres is equivalent to that observed in the SST, whereby the diaphragm opening process causes an increase in shock speed. For both conditions a dip is then observed in the velocity of the shock, after which there is a recovery to an approximately steady value by $5 \mathrm{~m}$. In comparison with the SST results of Fig. 8b, the scored diaphragms when used in the AST produce a flatter experimental shock speed profile. In the AST section, the maximum deviation from the mean shock speed is approximately $2 \%$ and $5 \%$ for Condition A and C respectively. This total variation is again low in the context of other similar facilities (Brandis et al. 2010a; Chandel et al. 2019).

Figure 9 also shows a comparison with both the PITOT and L1d 3 codes. A positive result is that in both cases the shock speed at the end of the tube exceeds the ideal PITOT prediction. This is due to the state-to-state code's current implementation of the nozzle expansion process, which assumes the gas upstream of the shock tube steadily expands completely through the nozzle by an amount based on the area ratio between the AST and Shock Tube 1. The unsteady expansion into the AST test gas, and by extension shock speed, is then calculated based on these ideally expanded conditions. In reality, it is likely that the unsteady and steady expansion processes occur somewhat concurrently. Higher-dimensional effects, such as waves 


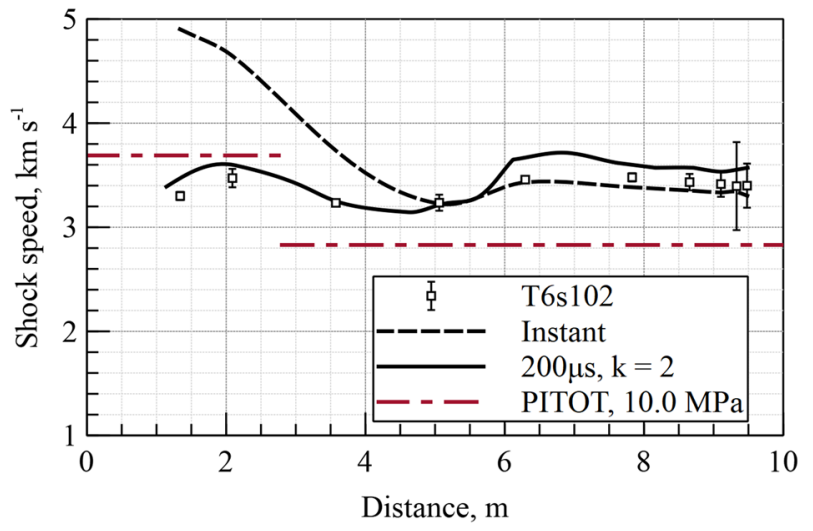

(a) Condition $\mathrm{A}, \mathrm{P}_{1}=26.6 \mathrm{~Pa}$.

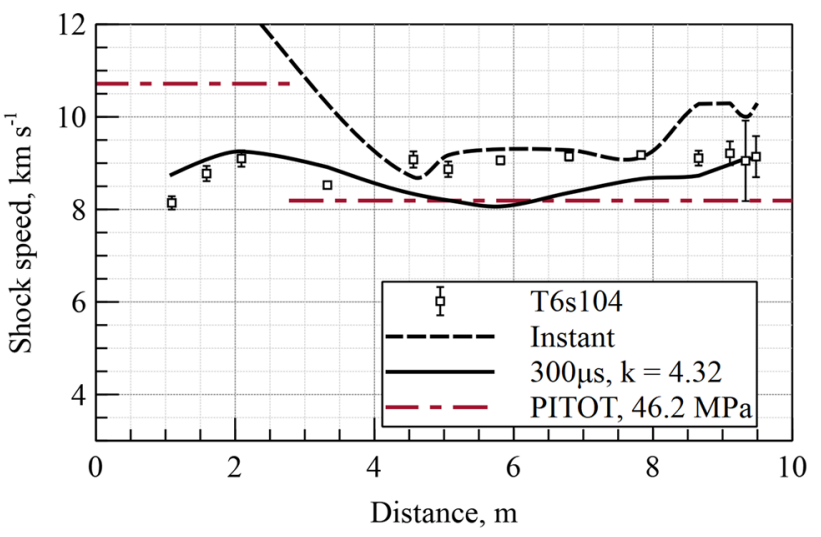

(b) Condition $\mathrm{C}, \mathrm{P}_{1}=26.6 \mathrm{~Pa}$.

Fig. 9 AST test data with comparison to numerical predictions. All tests were conducted in laboratory air

stemming from the driver, boundary layer growth and flow features in the nozzle may also moderate this behaviour.

Figure 9 also contains results of L1d 3 simulations for each condition. In both cases, the diaphragm opening conditions-as determined from the SST data in Fig. 8-were used. It can be seen that there is good agreement in the 96.3-mm tube (up to $3 \mathrm{~m}$ ) and in the ultimate shock speed at the end of the AST. The flow is generally well-predicted for the experiments at Condition A, including with the instantaneous opening model (after approximately $5 \mathrm{~m}$ ). However, for Condition C the finite opening time L1d3 results do not match the experimental data well in the region downstream of the nozzle. The agreement between $4 \mathrm{~m}$ and $8 \mathrm{~m} \mathrm{~m}$ is better with the instantaneous model, but the arrival of a strong compression wave (from the driver) at approximately $8 \mathrm{~m}$ causes divergence from the experimental data. This behaviour has found to be consistent for a wide range of simulated rupture pressure and diaphragm opening parameters. A higher-fidelity computational assessment of the flow is underway to better quantify the

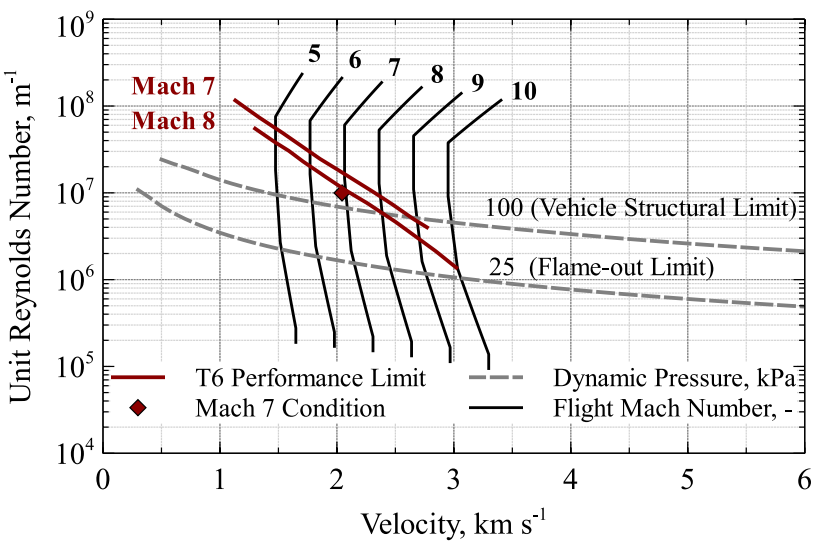

Fig. 10 Performance envelope of T6 in RST mode

nature of the wave processes through the nozzle and their influence on the shock speed downstream.

\section{Reflected shock tunnel mode}

Figure 10 shows a comparison between the performance envelope of T6 in RST mode and typical air-breathing flight corridors across a range of flight velocities. Iso-curves of Mach number and dynamic pressure are given as a function of flight velocity and unit Reynold's number, calculated using the US Standard Atmosphere Model (NASA 1976). At a given Mach number, the maximum and minimum unit Reynold's numbers are, respectively, defined by the structural strength of the vehicle and the ability to maintain combustion.

At the current time, contoured nozzles for nominal Mach numbers of 7 and 8 are available. Figure 10 also shows the maximum predicted performance envelope of T6 in RST mode with both of these nozzles. These performance curves have again been calculated using PITOT, using driver condition $\mathrm{C}$ (detailed in Table 2). An equilibrium expansion through the nozzle based on the ratio of the throat to exit diameters is also assumed. From these results, it can be seen that with the current infrastructure T6 is theoretically capable of reproducing the full air-breathing flight corridor up to Mach 8. Finally, Fig. 10 also shows the Mach 7 flight condition targeted in this work. The details of the measured properties at this test point are discussed in the subsequent sections.

\subsection{Reflected shock tunnel mode: experimental arrangement}

For the experimental data presented here, driver condition HA (as detailed in Table 2) was used. The shock tube was filled to a pressure of $180 \mathrm{kPa}$, using a synthetic air mixture 


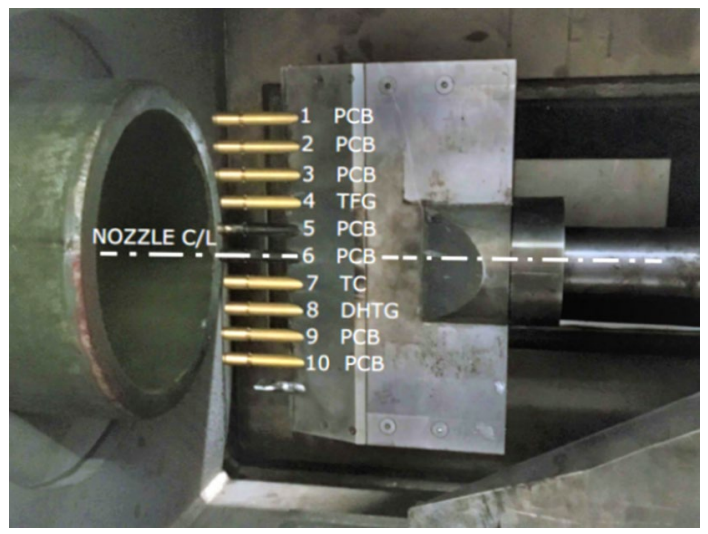

Fig. 11 Pitot rake in the T6 test section with gauge positions labelled (from Penty Garaets (2019))

of $79.2 \% \mathrm{~N} 2$ and $20.8 \% \mathrm{O} 2$ with total contaminants less than $2.2 \mathrm{ppm}$. This composition was used in preference to laboratory air to minimise errors in the thermochemical modelling of the nozzle discussed in Section 5.2.1. The analysis process used to select the facility filling conditions required for a desired test point has previously been presented Subiah et al. (2019).

As discussed in Section 2.2.2, the nozzle supply conditions in a reflected shock tunnel are generated by passing a strong shock through the test gas and then causing said gas to stagnate upstream of the throat. The total conditions are typically calculated as follows: the total pressure is measured directly using pressure transducers upstream of the throat. The total enthalpy is inferred using the ESTCj code of Jacobs et al. (2011), wherein the measured initial shock tube fill pressure and shock speed are used as inputs to calculate the conditions through an ideal incident/reflected shock process. The nozzle supply conditions are then isentropically relaxed to the measured stagnation pressure, with the total enthalpy of the test flow calculated from these relaxed values. Proper characterisation of the test flow thus requires accurate measurement of the stagnation pressure and the incident shock speed along the facility.

The shock speed measurements are performed identically to those described in Section 4.1. In the nozzle stagnation region, PCB Piezotronics 102B03 series pressure transducers are used to measure the pressure behind the reflected shock. For these experiments, a Pitot rake was positioned in the test section at a distance of $25 \mathrm{~mm}$ from the nozzle exit. As shown in Fig. 11, the rake consists of a steel wedge with 11 measurement positions, evenly spaced at $20-\mathrm{mm}$ intervals from the centreline. For these tests both Pitot pressure (using 7 PCB Piezotronics series 113B27 piezoelectric transducers) and stagnation point heat flux measurements were made. The latter employed a platinum thin-film gauge, a coaxial surface-junction thermocouple (James et al. 2019) and a novel

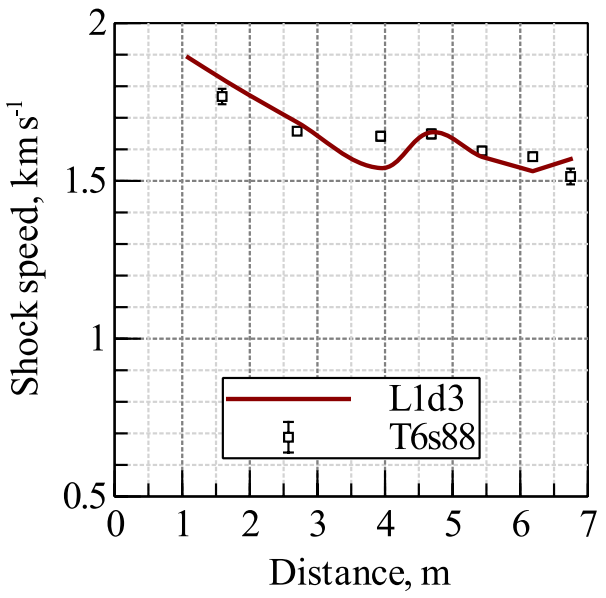

Fig. 12 Shock speed comparison between shot T6s88 and the L1d3 prediction

diamond calorimeter heat transfer gauge (DHTG) developed by Penty Geraets et al. (2020). All sensors are held within brass probe bodies which extend upstream of the rake. In the post-test flow, impacts by fragments of metallic diaphragm material often occur. Consequently, the pressure transducers were protected by shield caps as described by Neely et al. (1991). The heat transfer gauges must be exposed to the flow to enable accurate measurements and so were flushmounted in cylindrical bodies of diameter $10.50 \mathrm{~mm}$ and corner radius $0.13 \mathrm{~mm}$.

\subsection{Reflected shock tunnel mode: experimental results}

The measured shock speed variation through the facility is compared with an L1d3 prediction in Fig. 12. For the L1d3 simulations, the finite opening model presented in Section 4.3 was used, with the slow opening profile and opening time of $180 \mu$ s applied. Good agreement is generally observed between the measured shock speed and the L1d3 prediction, although the simulation predicts a sharper acceleration at approximately $4 \mathrm{~m}$. The reason for this feature can be identified in Fig. 13, which plots the results of the simulation as a distance-time diagram using the log of static pressure. Here, a compression wave-originating from the driver-is seen to reach the shock front at approximately 4 $\mathrm{m}$, which causes it to accelerate. This effect appears earlier in the experimental data, though its precise position is not resolved given the spacing of the shock timing stations. The observed discrepancy is likely due to the tunnel geometry and diaphragm rupture model used in the simulation, which significantly simplifies the wave transmission processes occurring in this region.

Based on the experimental data, the shock speed for this condition is taken to be $1.52 \mathrm{~km} \mathrm{~s}^{-1}$, which is the average 
Fig. 13 Distance-time diagram of the L1d3 simulation of shot T6s88. The time axis is referenced to the arrival of the incident shock at the nozzle supply transducer, located at approximately $7 \mathrm{~m}$



value of the final three measurements. Using ESTCj, this results in a predicted stagnation enthalpy of $2.68 \mathrm{MJ} \mathrm{kg}^{-1}$ which is within $2 \%$ of the target value of $2.7 \mathrm{MJ} \mathrm{kg}^{-1}$.

Figure 14 shows a comparison between the experimentally measured stagnation pressure and the numerical calculation. Considering the experimental trace, the stagnation pressure aligns well with the target value of $26 \mathrm{MPa}$. The test time at the nominal condition, defined as $\pm 10 \%$ of the target stagnation pressure value, is found from the experimental data to be $0.4 \mathrm{~ms}$. This is also marked in Fig. 14. Whilst this period represents the duration at the nominal condition, there is valid test flow after this period which can be analysed using quasi-steady methods before the onset of driver gas contamination (McGilvray et al. 2010).

In general, there is good agreement between $\mathrm{L} 1 \mathrm{~d} 3$ and the experimental results, both in terms of pressure magnitude during the test time and the decay rate. A sharp feature is present at approximately $1.2 \mathrm{~ms}$ in the simulated trace which is not observed in the experiment. The source of this wave is again visible in the distance-time diagram of Fig. 13, where the stagnation pressure measurement is made at approximately $7 \mathrm{~m}$. Here, the interaction of the reflected shock with the under-tailored contact surface generates a series of waves which travel downstream. These are significantly sharper in the computational result due to the idealised nature of the contact surface; in reality, the boundary between the driver and driven gases is highly three-dimensional (Goozée et al. 2006), which tends to smooth out strong pressure reflections. This is not captured in L1d3 due to the quasi-one-dimensional nature of the code; similar discrepancies have been seen in the simulation of other facilities (Mundt et al. 2007).

Whilst the L1d3 results show a clean rise between the incident and reflected shock conditions, the experimental trace contains an intermediate pressure step. A similar feature is observed in some-but not all-other RST facilities and is not captured in other studies using L1d3 (Mundt et al. 2007). A full-facility numerical study of a reflected shock tunnel by Goozée et al. (2006) showed this feature to be caused by a complex interaction between the non-planar reflection of the shock from the end wall and the boundary layer, resulting in shock bifurcation. Further results presented in the thesis of Goozee (2003) using a combined experimental-numerical approach showed this pressure step to be significantly affected by the boundary layer thickness (and hence Reynolds number) and the end-wall geometry used. These factors explain both the inconsistencies in observation between facilities and why the (quasione-dimensional) L1d 3 code does not reproduce the feature. Higher-fidelity simulations to characterise this behaviour for

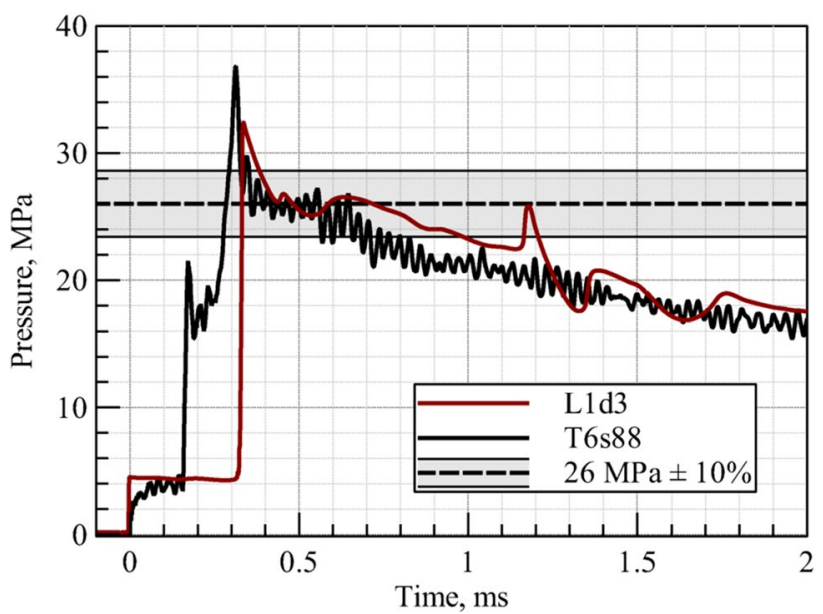

Fig. 14 Nozzle supply pressure comparison between shot T6s 88 and L1d3 prediction 


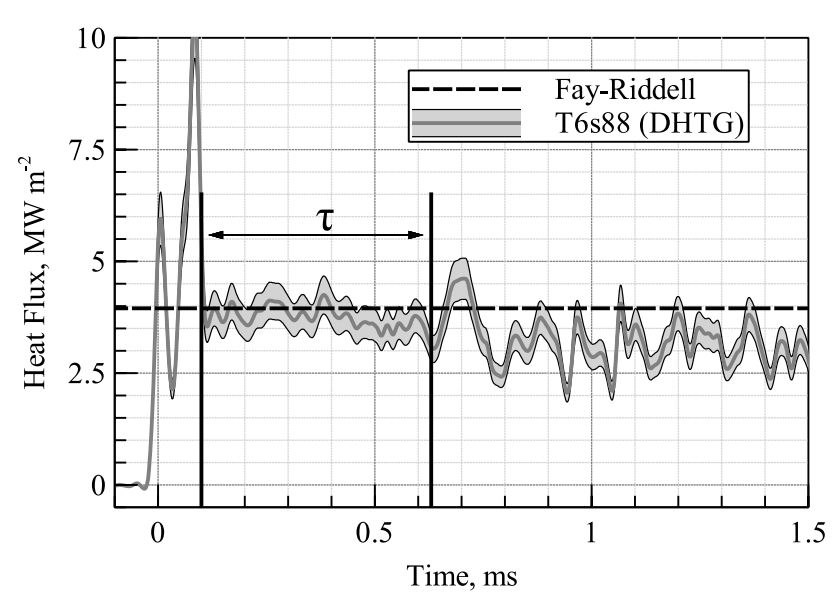

Fig. 15 Stagnation-point heat flux using the DHTG compared to the analytic calculation. The identified period of quasi-steady test flow is denoted by $\tau$

the specific geometric case of the T6 RST mode are currently on-going.

Using the ESTCj code, the total enthalpy was calculated to be $2.68 \mathrm{MJ} \mathrm{kg}^{-1}$. Knowing the total pressure and enthalpy of the test condition, the method of Fay and Riddell (1958) was used to estimate the expected stagnation point heat flux on a spherical body. As discussed in Section 5.1, the heat flux gauge holders are cylindrical; an empirical correction for an 'effective radius' (Zoby and Sullivan 1966)_giving a value of $15.21 \mathrm{~mm}$ - has thus been used in the theoretical calculation.

The calculated heat flux for this condition is compared in Fig. 15 with the experimental result from the DHTG. Good agreement is observed between the experimental heat flux measurements and the Fay-Riddell prediction during the test time. The measurement uncertainty for the DHTG is given by Penty Geraets et al. (2020) as 10\%, shown as the shaded region in Fig. 15. This close agreement builds confidence in the calculated value of total enthalpy which will be further confirmed with direct measurements of total temperature via optical techniques in future experiments.

\subsubsection{Reflected shock tunnel mode: nozzle flow analysis}

To estimate additional properties of the test flow, a computational study was undertaken using an open-source Reynolds-averaged Navier--Stokes computational fluid dynamics solver, eilmer4 (Jacobs and Gollan 2016). The approach taken was identical to that used in previous studies of similar facilities, e.g. Chan et al. (2018), where the nozzle is simulated from the throat onwards. The measured stagnation properties are expanded to a Mach number of 1 to give a uniform inflow at the throat. The wall of the nozzle was assumed to be at a constant temperature of $300 \mathrm{~K}$. A

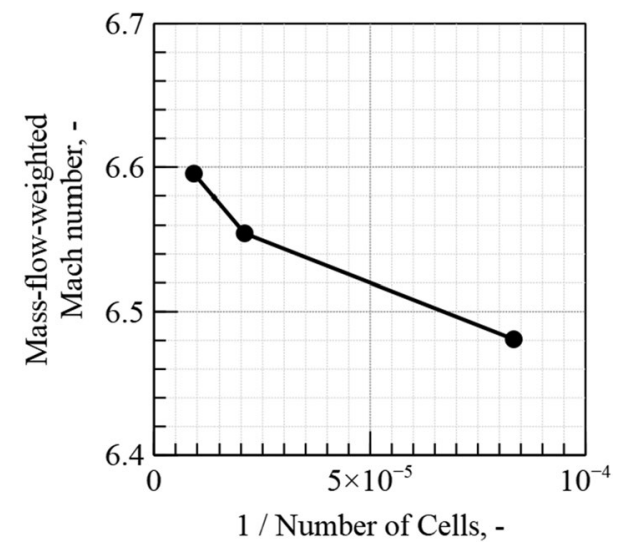

Fig. 16 Grid convergence analysis based on the mass-flow-weighted Mach number

pseudo-space-marching approach were used to reduce the computational time needed to obtain a steady-state nozzle flow solution. The turbulence model used in the simulations was the k- $\omega$ turbulence model of Wilcox et al. (1998). To further reduce computational time, the wall functions of Nichols and Nelson (2004) was used to model the turbulent boundary layers. The application of wall functions is appropriate since boundary layer separation is not expected in the nozzle flow field. The turbulence intensity of the inflow to the nozzle was set to $5 \%$, with a ratio of the turbulent-tolaminar viscosity of 100 . To examine the influence of boundary layer transition location on the core flow parameters, simulations with different transition locations downstream of the nozzle throat were performed. The boundary layer transition location was varied until a satisfactory agreement with the experimental Pitot pressure data was achieved. The final transition location is $380-\mathrm{mm}$ downstream of the nozzle throat.

The computational grid which produced converged solutions for the flow simulations had 600 cells in the axial direction and 80 cells in the radial direction. Grid clustering was employed near the throat and initial expansion regions to resolve the larger flow gradients expected in these regions and near the nozzle wall to ensure adequate resolution of the boundary layer. The grids near the nozzle wall were configured to be orthogonal to the wall to ensure that the near-wall flow parameters were properly approximated by the flow solver. The level of grid convergence is shown in Fig. 16 with a plot of the mass-flow-weighted Mach number plotted against the inverse of the total cell number for each grid resolution. The coarse grid had 300 axial cells and 40 radial cells, the medium grid had 600 axial cells and 80 radial cells, whilst the fine grid had 900 axial cells and 120 radial cells. The mass-flow-weighted Mach number for the medium grid differed from the fine grid by only $0.7 \%$. It was thus concluded that the medium grid produced a flow field 
Fig. 17 Contour plot of the Mach number distribution for the T6 RST Mach 7 nozzle and test section flow field. The solid white lines indicate the core flow region. The white dasheddot line shows the position of the Pitot rake

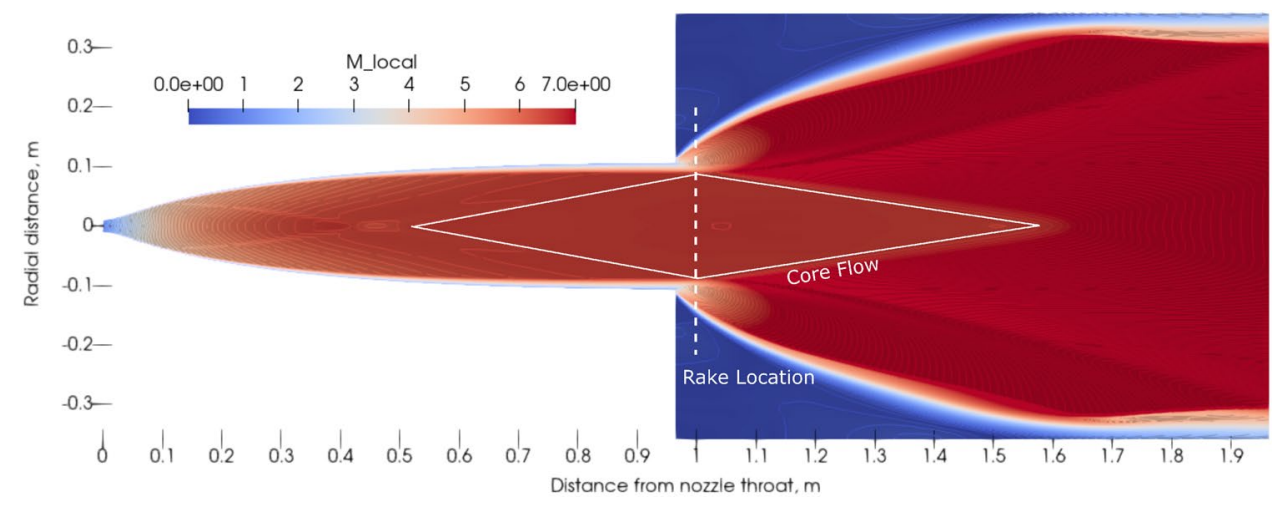

at the exit of the nozzle that was sufficiently grid-converged for the analysis conducted in the present study.

The simulations were performed with three different thermo-chemical gas models to investigate the influence of high enthalpy chemical kinetics. These models were: equilibrium thermo-chemistry based on the 5-species air model from the CEA2 program of Gordon and McBride (1994), finite-rate chemistry based the Gupta and Thompson (1989) 5-species air model and two-temperature thermochemistry based on the 2-temperature, 5-species air model of Park (1989). The two temperatures considered are the translational-rotational and vibrational-electronic modes; it is assumed that these quantities represent the internal energy distribution for all chemical species. These thermo-chemistry models are referred to in this section of the paper as the equilibrium, chemical non-equilibrium and thermo-chemical non-equilibrium models, respectively. The mixture transport properties (i.e. mixture viscosity and thermal conductivity) were calculated using mixing rules of Gordon and McBride (1994) which are a variant of Wilke's original formulation (Wilke 1950).

Figure 17 presents a contour plot of Mach number isolines that were generated from simulations of the Mach 7 nozzle and the test section of the T6 facility using the equilibrium model. The test section flow field was calculated separately, using the conditions from the nozzle simulation at a distance of $27.9 \mathrm{~mm}$ upstream of the exit as an inflow. The location of the Pitot rake is shown by a vertical dashdot line and the bi-conic core-flow region by a dashed line. This region has a maximum axial length of $1050 \mathrm{~mm}$ and a maximum diameter of $180 \mathrm{~mm}$.

A comparison of the experimental and simulated Pitot pressure at the measurement location is provided in Fig. 18. The experimental values shown are the average Pitot pressure during the test time, normalised by the mean nozzle supply pressure over the same period. The results show good uniformity across the core flow region, which the experimental data suggests has a diameter of at least $160 \mathrm{~mm}$. Also shown in Fig. 18 are the Pitot pressure results from the three thermo-chemical models. To calculate these values, the flow over a simplified Pitot probe geometry was simulated using nozzle exit conditions for each of the three thermochemical cases. Figure 18 shows an excellent match between the experiments and the simulations, and that there is a good level of Pitot pressure uniformity in the core flow.

It is important to note that whilst the different thermochemistry models have little effect on the Pitot pressures (as shown in the comparison of Fig. 18), they do have more significant influence on the free-stream flow propertiesplots of nozzle-exit Mach number and static pressure from the three thermo-chemical simulations in Fig. 19 reveal this. Whilst the equilibrium and chemical non-equilibrium cases result in very small differences in Mach number and static pressure, the results for the thermo-chemical non-equilibrium model display significant departure from the other two models. The free-stream unit Reynold's number ranges from $8.9 \times 10^{6} \mathrm{~m}^{-} 1$ for the thermo-chemical non-equilibrium simulation to $9.3 \times 10^{6} \mathrm{~m}^{-} 1$ for the chemical non-equilibrium model.

Finally, Fig. 20 shows (a) differences in temperature for the three thermo-chemistry models and (b) the amount of vibrational energy freezing for the thermo-chemical

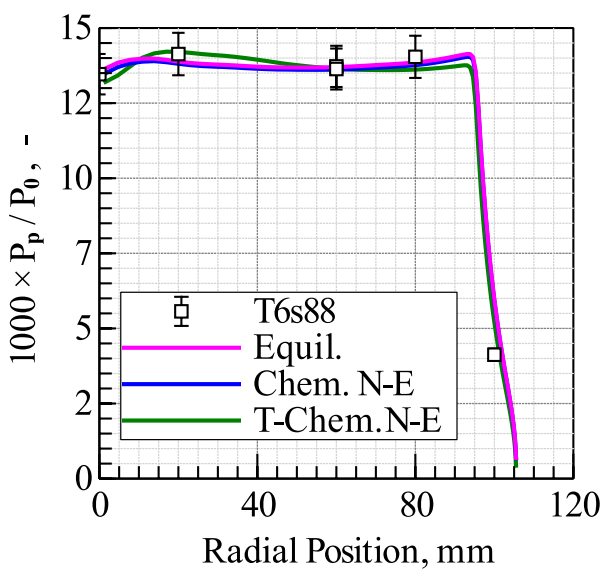

Fig. 18 Pitot-to-total pressure ratio comparison between shot T6s88 and axisymmetric CFD model 


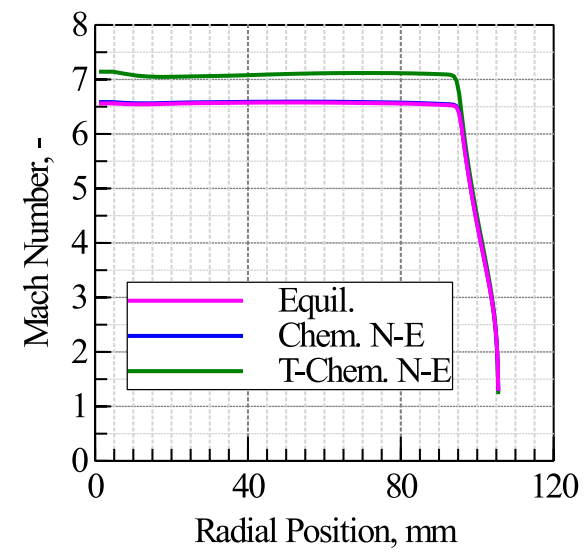

(a) Mach number variation at nozzle exit.



(b) Static pressure variation at nozzle exit.

Fig. 19 Radial profiles of nozzle exit Mach number and static pressure

non-equilibrium solution. These simulations reveal that the free-stream static temperature is approximately $270 \mathrm{~K}$ in the case of the equilibrium and chemical non-equilibrium simulations, as well as for the thermo-chemical non-equilibrium translational mode. In contrast, at nozzle exit the vibrational temperature is frozen at $1270 \mathrm{~K}$; for context, the static temperature at the nozzle throat is $2100 \mathrm{~K}$.

The obtained nozzle exit flow properties display significant departure from the equilibrium, highlighting the need to take the influence of finite-rate thermo-chemistry into account when simulating hypersonic nozzle flows. These results show that these non-equilibrium effects are significant at the presented condition. This aligns with results from other RST facilities at similar enthalpies, for example Hannemann et al. (2010). Future experiments are planned to confirm the degree of thermal non-equilibrium in the flow field, both through optical techniques and measurement of the free-stream static pressure. Based on the current data,

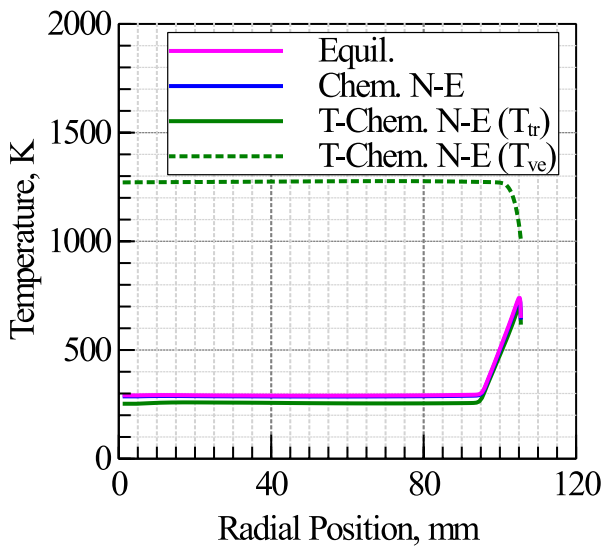

Fig. 20 Comparison of nozzle exit temperature distribution for the three thermo-chemistry models

good agreement is found between the targeted condition and the experimental results.

\section{Expansion tube mode}

The commissioning tests of T6 in ExT mode reported here focused on high-speed Earth return conditions. Both ESA and NASA are currently considering proposals for a Mars Sample Return (MSR) mission, a key challenge of which is the return of a sample capsule from Mars to Earth (Potter 2020). Given that any gathered material must be returned from Mars, minimisation of vehicle mass to increase possible payload - through reduction of thermal protection system size-is desirable. The return section of the mission will likely occur at very high speed, with potential entry velocities up to $22 \mathrm{~km} \mathrm{~s}^{-1}$ (Johnston and Brandis 2015). At these velocities, the post-shock temperature can significantly exceed the limits of many of the thermochemical models used to predict vehicle heating. Johnston and Brandis (2015) previously provided significant extensions to some of these models to enable their use for MSR trajectories. However, relevant ground test data will be required to validate predictions of the aerothermodynamic heating environment.

\subsection{Expansion tube mode: performance envelope}

The performance envelope of T6 in ExT mode in the context of MSR missions is shown in Fig. 21. Figure 21a shows a likely bounding region for MSR flight trajectories. Overlaid is the capability map of T6 in expansion tube mode, employing the current highest performance driver condition, C. Fig. $21 \mathrm{~b}$ shows a more detailed representation of T6 performance prediction, wherein iso-curves of shock tube fill pressure $\left(P_{1}\right)$ and acceleration tube pressure $\left(P_{5}\right)$ are shown. This performance prediction has again been produced using 
Fig. 21 Comparison of proposed MSR trajectories with T6 performance capability in ExT mode

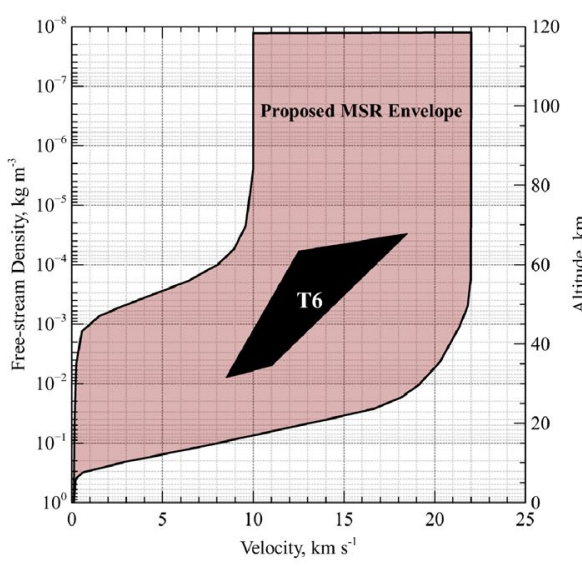

(a) Possible MSR trajectory envelope (adapted from Johnston and Brandis (2015)) with T6 performance prediction.

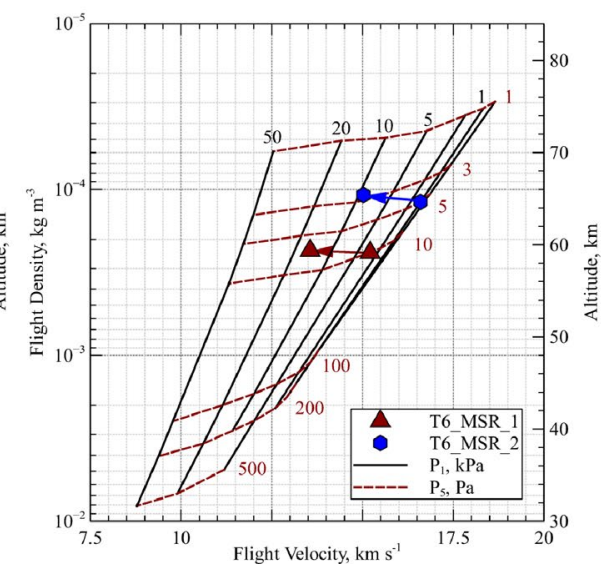

(b) Detailed view of T6 performance capability region, showing tested conditions.
PITOT, which has been extensively used for modelling of other expansion tube facilities (James et al. 2018).

It can be seen that the nominal capability of T6 lies in the centre of the map of possible trajectories for MSR missions. For stagnation point heat flux measurements the binary scaling parameter (the product of free-stream density and vehicle characteristic length scale) and flow total enthalpy are typically matched to flight to recreate the post-shock environment. The scaling in Fig. 21 assumes a 35-mm-diameter model is used with the density adjusted to match the conditions for a 900-mm-diameter flight vehicle, corresponding to a proposed Earth Entry Vehicle (EEV) design (Dillman and Corliss 2008). The model size is currently limited by the bore of the expansion tube $(96.3 \mathrm{~mm})$. Work is underway to design a nozzle, permitting an increase in outlet diameter to approximately $280 \mathrm{~mm}$ and thereby allowing significantly larger models to be tested.

\subsection{Expansion tube mode: experimental arrangement}

To validate the presented performance predictions an experimental study was undertaken in ExT mode. For the experiments detailed in this work, nominal shock tube pressures of $1 \mathrm{kPa}$ and $3 \mathrm{kPa}$ with acceleration tube pressures of $5 \mathrm{~Pa}$ and $10 \mathrm{~Pa}$ were used, respectively. The Pitot rake described in Section 5.1 was located in the test section, placed one tube radius from the tube exit. Due to the smaller core flow diameter and higher pressures in ExT mode, two PCB Piezotronic 104 series piezoelectric pressure transducers were used for these tests. These were again protected using the shield cap design of Neely et al. (1991). For all experiments an aluminium foil secondary diaphragm with measured rupture pressure of $15 \mathrm{kPa}$ was used.

\subsection{Expansion tube mode: experimental results}

The target test points, as well as the conditions achieved, are shown in Fig. 21b. Here, the arrows denote the shift in the velocity and free-stream density from prediction to experiment; reasons for this difference are discussed below. Also note that, based on Mirels (1963) analysis, the actual velocity of the expanded test gas in the laboratory frame of reference is effectively the acceleration tube shock speed. In comparison, the velocity plotted in Figure $21 \mathrm{~b}$ represents the 'flight-equivalent velocity' which accounts for the remaining static enthalpy of the test gas and is calculated by matching the experimental total enthalpy with flight.

Experimental results from the two conditions shown in Fig. $21 \mathrm{~b}$ are given in Fig. 22. Figures 22a and 22b show the measured shock speed variation along the length of the facility, with comparison to PITOT calculations. Note that the secondary diaphragm is located at approximately $2.8 \mathrm{~m}$. In general, it can be seen that PITOT over-predicts the achieved shock speed; this is to be expected given the idealisations in the code. As discussed in Section 4, the over-prediction in the shock tube is mostly due to diaphragm opening effects. A proportion of the test gas in the shock tube is thus processed by a weaker shock than expected, in effect reducing the performance of the driver. The second source of over-prediction by PITOT is that the code does not capture viscous or pressure losses. Notably, any loss through the secondary diaphragm - which is typically one of the largest performance loss mechanisms in an expansion tube-is not modelled. The mass of the secondary diaphragm is the main cause of this loss, and so ideally the lightest practicable ${ }^{2}$ material is used.

Figures $22 \mathrm{c}$ and $22 \mathrm{~d}$ show the measured Pitot pressure for each condition. Also shown in each figure is the value

\footnotetext{
$\overline{2}$ i.e. will not burst during facility evacuation or filling.
} 
Fig. 22 Experimental results for MSR expansion tube conditions. The identified region of quasi-steady test flow is denoted by $\tau$

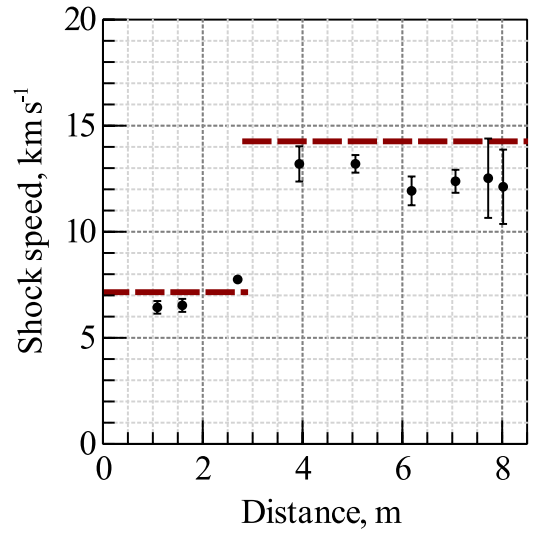

(a) T6-MSR-1 shock speeds.

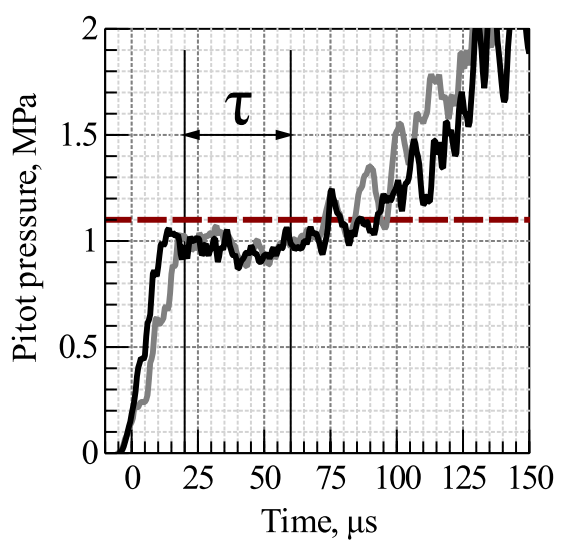

(c) T6-MSR-1 Pitot pressure.



(b) T6-MSR-2 shock speeds.

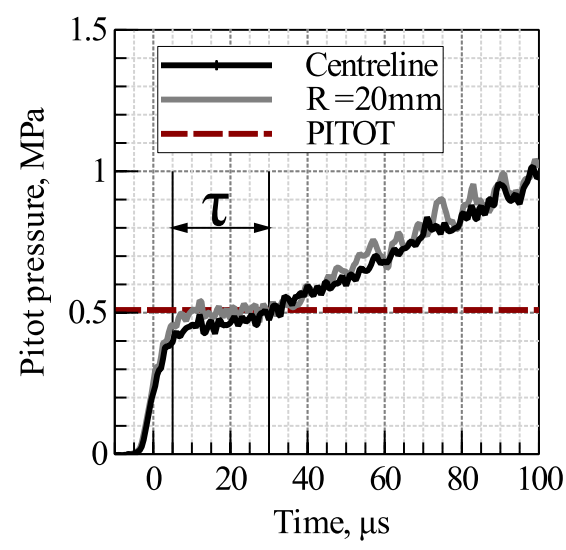

(d) T6-MSR-2 Pitot pressure.
Table 4 Flow properties of Mars Sample Return ExT conditions

\begin{tabular}{lllllll}
\hline Condition & $\mathrm{P}_{1}, \mathrm{kPa}$ & $\mathrm{P}_{5}, \mathrm{~Pa}$ & $\mathrm{P}_{0}, \mathrm{GPa}$ & $\mathrm{H}_{0}, \mathrm{MJ} \mathrm{kg}^{-1}$ & $\mathrm{U}_{\text {eq }}, \mathrm{km} \mathrm{s}^{-1}$ & $\tau, \mu \mathrm{s}$ \\
\hline T6-MSR-1 & 3 & 10 & 12.8 & 88.9 & 13.3 & 40 \\
T6-MSR-2 & 1 & 5 & 14.1 & 117.6 & 15.3 & 25 \\
\hline
\end{tabular}

from the PITOT code, where the experimentally measured shock speeds and fill pressures are used to calculate the flow conditions. For both conditions good agreement is found between the experimental traces and the predicted Pitot pressure. Referring to the distance-time diagram of Fig. 4d, it is expected that the Pitot trace should show first the arrival of the accelerator shock and gas, followed by the test gas and finally the unsteady expansion fan. The accelerator gas is not clearly observed in the Pitot pressure traces due to the filling time of the sensor housing (approximately $10 \mu \mathrm{s}$ ). Whilst a full characterisation of the flow field at the tube outlet has not yet been undertaken, these results suggest that the core flow diameter at this axial position is at a minimum $40 \mathrm{~mm}$ (based on the probe spacing). This implies that the choice of a $35-\mathrm{mm}$ model for the performance scaling of Fig. 21 is well within the limits required for the analysis to be valid. In both cases, the pressures show good steadiness throughout the test time.

Table 4 shows the test flow properties for both of the conditions presented; these are taken from PITOT, again with the experimentally measured pressure and shock speed used as inputs. The test time is determined based on the period observed before the Pitot pressure traces begin to trend upwards; this is associated with the arrival of the unsteady expansion and hence the end of the test time. Although short in absolute terms, the determined test times are sufficient to establish the thermal boundary layer based on the work of Davies and Bernstein (1969). From these results, it can be seen that the ExT mode has achieved flow conditions in excess of $15 \mathrm{~km} \mathrm{~s}^{-1}$ with highquality test flows of sufficient duration for aerothermodynamic ground testing. The experiments presented here thus 
demonstrate the capability of $\mathrm{T} 6$ to achieve hyperbolic atmospheric entry conditions.

\section{Conclusion}

This work has presented the development work to date on a new high-enthalpy, aerothermodynamic ground test facility-the T6 Stalker Tunnel. The facility is a combination of the original T3 free-piston driver, the Oxford Gun Tunnel barrels and newly designed components. Commissioning of the driver was first discussed, incorporating results using two pistons at nominal rupture pressures up to $46 \mathrm{MPa}$. Efforts to match a computational model of the driver to the experimental data were successful, with good agreement between the 'blank-off' test data and the model predictions shown.

Theoretical performance envelopes and experimental data from four distinct facility modes were then presented: reflected shock tunnel, shock tube, expansion tube and aluminium shock tube. In general, departures from the ideal predictions were attributed to 'real-world' effects such as diaphragm opening and the presence of wall boundary layers. Good agreement with the experimental data and higher-fidelity simulations of the facility was found. Notably, consideration of diaphragm opening effects and nonequilibrium thermo-chemistry gave close agreement to the measurements in the ST and RST modes. In all cases, high-quality test flows were achieved.

The demonstrated flow conditions cover a wide range of mission archetypes and physical regimes, from recreating high-speed atmospheric flight to hyperbolic Earth entries. Future work will further extend capability, for example through construction of additional nozzles for RST and ExT modes and construction of a lighter piston. Extension and more detailed characterisation of flow conditions are also on-going. Of particular note is that this work presents the first European capability for super-orbital shock tube experiments-embodied in the T6 SST and AST modes. In addition, the ability to achieve MSR conditions in a ground test facility has also been demonstrated through expansion tube operation. These achievements highlight the unique value of T6 to support next-generation space missions through the provision of ground test data.

Acknowledgements Gratitude is extended to Peter Jacobs, Chris James and all at the University of Queensland for their support in the design and commissioning of the facility. We are eternally grateful for the contributions of the Oxford Thermofluids Institute Workshop and Building Services teams, with particular thanks owed to William Godfrey, Hal Surtell and James Carter for their substantial efforts expended over the course of making T6 operational.
Funding Sources The authors would like to acknowledge the UK National Wind Tunnel Facility for their support in the development of the T6 Stalker Tunnel under grant EP/L024888/1. Thanks also to the Oxford University John Fell Fund and Fluid Gravity Engineering for their support in the development of the facility.

Open Access This article is licensed under a Creative Commons Attribution 4.0 International License, which permits use, sharing, adaptation, distribution and reproduction in any medium or format, as long as you give appropriate credit to the original author(s) and the source, provide a link to the Creative Commons licence, and indicate if changes were made. The images or other third party material in this article are included in the article's Creative Commons licence, unless indicated otherwise in a credit line to the material. If material is not included in the article's Creative Commons licence and your intended use is not permitted by statutory regulation or exceeds the permitted use, you will need to obtain permission directly from the copyright holder. To view a copy of this licence, visit http://creativecommons.org/licenses/by/4.0/.

\section{References}

Andrianatos A, Gildfind D, Morgan R (2015) A study of radiation scaling of high enthalpy flows in expansion tubes. In: 7th Asia Pacific International Symposium on Aerospace Technology

Andrianatos A, Gildfind D Morgan R (2016) Preliminary development of high enthalpy conditions for the X3 Expansion Tube. In: 20th Australasian Fluid Mechanics Conference

Boyer AG (1965) Design, instrumentation and performance of the UTIAS 4-in. x 7-in. hypersonic shock tube. Technical Report 99, Institute for Aerospace Studies, University of Toronto

Brandis A, Cruden B, Prabhu D, Bose D, McGilvray M, Morgan R (2010a) Analysis of air radiation measurements obtained in the EAST and X2 shocktube facilities. In: 10th AIAA/ASME Joint Thermophysics and Heat Transfer Conference

Brandis AM, Cruden BA (2017a) Benchmark shock tube experiments of radiative heating relevant to Earth re-entry. In: 55th AIAA Aerospace Sciences Meeting

Brandis AM, Cruden BA (2017b) Titan atmospheric entry radiative heating. In: 47th AIAA Thermophysics Conference

Brandis AM, Johnston CO (2014) Characterization of stagnation-point heat flux for Earth entry. In: 45th AIAA Plasmadynamics and Lasers Conference

Brandis AM, Morgan RG, McIntyre TJ, Jacobs PA (2010b) Nonequilibrium radiation intensity measurements in simulated Titan atmospheres. J Thermophys Heat Trans 24(2):291-300

Chan WY, Jacobs PA, Smart MK, Grieve S, Craddock CS, Doherty LJ (2018) Aerodynamic design of nozzles with uniform outflow for hypervelocity ground-test facilities. J Propulsion Power 34(6): 1467-1478

Chandel D, Nompelis I, Candler GV, Brandis AM (2019) CFD predictions of high enthalpy shocks in nitrogen. In: AIAA Aviation 2019 Forum, page 3078

Collen PL, Doherty LJ, McGilvray M (2019) Measurements of radiating hypervelocity air shock layers in the T6 Free-Piston Driven Shock Tube. In: International Conference on Flight Vehicles, Aerothermodynamics and Re-entry Missions \& Engineering. ESA Conference Bureau

Cruden B (2012) Radiance measurement for low density Mars entry. In: 43rd AIAA Thermophysics Conference. p page 2742

Cruden BA, Brandis AM, Prabhu DK (2014) Measurement and characterization of mid-wave infrared radiation in $\mathrm{CO} 2$ shocks. In: 11th AIAA/ASME Joint Thermophysics and Heat Transfer Conference, page 2962 
Davies W, Bernstein L (1969) Heat transfer and transition to turbulence in the shock-induced boundary layer on a semi-infinite flat plate. J Fluid Mech 36(1):87-112

Dillman R, Corliss J (2008) Overview of the Mars Sample Return Earth Entry Vehicle. In: Sixth International Planetary Probe Workshop

Doherty LJ, Smart MK, Mee DJ (2015) Experimental testing of an airframe-integrated three-dimensional scramjet at Mach 10. AIAA J 53(11):3196-3207

Doolabh A (2016) A concept study for a piston driven sCO2 turbine test facility. University of Queensland, Queensland (Dissertation)

Doolan C, Jacobs P (1996) Modeling mass entrainment in a quasi-onedimensional shock tube code. AIAA J 34(6):1291-1293

Dufrene A, Sharma M, Austin J (2007) Design and characterization of a hypervelocity expansion tube facility. J Propuls Power 23(6):1185-1193

Fay JA, Riddell FR (1958) Theory of stagnation point heat transfer in dissociated air. J Aerospace Sci 25(2):73-85

Fletcher DG (2004) Fundamentals of hypersonic flow - aerothermodynamics. Lecture Series on Critical Technologies for Hypersonic Vehicle Development, Von Karman Institute, Rhode-St-Genise, Belgium, pp 10-14

Gildfind DE (2012) Development of high total pressure scramjet flow conditions using the X2 expansion tube. University of Queensland, $\mathrm{PhD}$ thesis

Gildfind DE, Jacobs PA, Morgan RG (2013) Vibration isolation in a free-piston driven expansion tube facility. Shock Waves 23:431-438

Gildfind DE, James CM, Toniato P, Morgan RG (2015) Performance considerations for expansion tube operation with a shock-heated secondary driver. J Fluid Mech 777:364-407

Gildfind DE, Morgan RG, Jacobs PA (2016) Expansion tubes in Australia. Experimental Methods of Shock Wave Research. Springer, pp 399-431

Goozee RJ (2003) Simulation of a complete shock tunnel with parallel computer codes. The University of Queensland, $\mathrm{PhD}$ thesis

Goozée RJ, Jacobs PA, Buttsworth DR (2006) Simulation of a complete reflected shock tunnel showing a vortex mechanism for flow contamination. Shock Waves 15(3-4):165-176

Gordon S, McBride BJ (1994) Computer program for calculation of complex chemical equilibrium compositions and applications: I. analysis. NASA Reference Report NASA RP-1311, NASA Lewis Research Center, Cleveland, $\mathrm{OH}$

Grinstead JH, Wilder MC, Reda DC, Cornelison CJ, Cruden BA, Bogdanoff DW (2010) Shock tube and ballistic range facilities at NASA Ames Research Center. Technical report. NATO RTO-EN-AVT-186

Gupta RN, Thompson RA (1989) A review of reaction rates and thermodynamic and transport properties for the 11-species air model for chemical and thermal non-equilibrium calculations to 30000 K. NASA Tech Memorandum 101:528

Hannemann K, Itoh K, Mee DJ, Hornung HG (2016) Free Piston Shock Tunnels HEG, HIEST, T4 and T5. Experimental Methods of Shock Wave Research. Springer, pp 181-264

Hannemann K, Schramm JM, Wagner A, Karl S, Hannemann V (2010) A closely coupled experimental and numerical approach for hypersonic and high enthalpy flow investigations utilising the HEG Shock Tunnel and the DLR TAU code. Technical report, German Aerospace Centre Braunschweig

Holden MS, Wadhams TP, MacLean M (2008) Experimental studies in the LENS supersonic and hypersonic tunnels for hypervelocity vehicle performance and code validation. In: 15th AIAA International Space Planes and Hypersonic Systems and Technologies Conference
Hornung H (1990) Role and techniques of ground testing for simulation of flows up to orbital speed. In: 16th Aerodynamic Ground Testing Conference, page 1377

Itoh K, Ueda S, Komuro T, Sato K, Takahashi M, Miyajima H, Tanno $\mathrm{H}$, Muramoto H (1998) Improvement of a free piston driver for a high-enthalpy shock tunnel. Shock Waves 8(4):215-233

Jacobs C, McIntyre T, Morgan R, Brandis A, Laux C (2015) Radiative heat transfer measurements in low-density Titan atmospheres. J Thermophys Heat Trans 29(4):835-844

Jacobs P, Gollan R (2016) Implementation of a compressible-flow simulation code in the D programming language. Appl Mech Mater 846:54-60

Jacobs P, Gollan R, Potter D, Zander F, Gildfind D, Blyton P, Chan W, Doherty L (2011) Estimation of high-enthalpy flow conditions for simple shock and expansion processes using the ESTCj program and library. University of Queensland, Queensland (Technical report)

Jacobs PA (1998) Shock tube modelling with L1d. Research Report 13/1998, The University of Queensland, Department of Mechanical Engineering, Brisbane, Australia

James C, Gildfind D, Lewis S, Morgan R, Zander F (2018) Implementation of a state-to-state analytical framework for the calculation of expansion tube flow properties. Shock Waves 28(2):349-377

James CM (2019) Using a simplified Canny edge detector to detect shock arrival in expansion tubes and shock tunnels. In: Proceedings of the 32nd International Symposium on Shock Waves

James CM, Birch B, Smith DR, Cullen TG, Millard T, Vella S, Liu Y, Morgan RG, Stern N, Buttsworth D (2019) Testing of ultra fast response, durable co-axial thermocouples for high enthalpy impulse facilities. In: AIAA Aviation 2019 Forum, page 3007

Johnston CO, Brandis AM (2015) Aerothermodynamic characteristics of $16-22 \mathrm{~km} / \mathrm{s}$ Earth entry. In: 45th AIAA Thermophysics Conference, page 3110

Lu FK, Marren DE, editors (2002) Advanced Hypersonic Test Facilities, volume 198 of Progress in Astronautics and Aeronautics. American Institute of Aeronautics and Astronautics

MacLean M, Dufrene A, Holden M (2013) Spherical capsule heating in high enthalpy carbon dioxide in LENS-XX expansion tunnel. In: 51st AIAA Aerospace Sciences Meeting including the New Horizons Forum and Aerospace Exposition, page 906

MacLean M, Holden M (2006) Numerical assessment of data in catalytic and transitional flows for Martian entry. In: 9th AIAA/ASME Joint Thermophysics and Heat Transfer Conference, page 2946

McGilvray M, Doherty LJ, Morgan RG, Gildfind D, Jacobs P, Ireland P (2015) T6: The Oxford University Stalker tunnel. In: 20th AIAA International Space Planes and Hypersonic Systems and Technologies Conference

McGilvray M, Morgan RG, Jacobs PA (2010) Scramjet experiments in an expansion tunnel: Evaluated using a quasi-steady analysis technique. AIAA J 48(8):1635-1646

Mirels H (1963) Test time in low-pressure shock tubes. Phys Fluids 6(9):1201-1214

Mirels H (1964) Shock tube test time limitation due to turbulent-wall boundary layer. AIAA J 2(1):84-93

Mundt C (2016) Development of the new piston-driven shock-tunnel HELM. Experimental Methods of Shock Wave Research. Springer, pp 265-283

Mundt C, Boyce RR, Jacobs PA, Hannemann K (2007) Validation study of numerical simulations by comparison to measurements in piston-driven shock-tunnels. Aerospace Sci Technol 11(2-3):100-109

NASA (1976). U.S. Standard Atmosphere 1976. Technical Memorandum $x-74335$, NASA 
Neely AJ, Stalker RJ, Paull A (1991) Hypervelocity flows of argon produced in a free piston driven expansion tube. In: Shock Waves, volume 2, pages 997-1004. Springer Verlag

Nichols RH, Nelson C (2004) Wall function boundary conditions including heat transfer and compressibility. AIAA J 42(6):1107-1114

Options for enhancement of the performance of shock-expansion tubes and tunnels. In: 33rd Aerospace Sciences Meeting and Exhibit

Park C (1989) Assessment of two-temperature kinetic model for ionizing air. J Thermophys Heat Trans 3(3):233-244

Penty Garaets R (2019) Development and testing of a diamond calorimeter heat transfer gauge. University of Oxford, $\mathrm{PhD}$ thesis

Penty Geraets RT, McGilvray M, Doherty LJ, Morgan RG, James CM, Buttsworth DR (2020) Development of a fast-response diamond calorimeter heat transfer gauge. J Thermophys Heat Trans 34(1):193-202

Potter S (2020) NASA moves forward with campaign to return Mars samples to Earth. https://www.nasa.gov/press-release/nasamoves-forward-with-campaign-to-return-mars-samples-to-earth. accessed 29/12/20

Rothkopf E, Low W (1974) Diaphragm opening process in shock tubes. Physics Fluids 17(6):1169-1173

Satchell M, Collen P, McGilvray M, Di Mare L (2021) Numerical simulation of shock tubes using shock tracking in an overset formulation. AIAA Journal 1-11

Sharma SP, Park C (1990) Operating characteristics of a 60-and 10 -cm electric arc-driven shock tube. J Thermophys Heat Trans 4(3):259-265

Simpson C, Chandler T, Bridgman K (1967) Effect on shock trajectory of the opening time of diaphragms in a shock tube. Phys Fluids 10(9):1894-1896

Stalker R (1972) Development of a hypervelocity wind tunnel. Aeronautic J 76(738):374-384

Stalker R, Crane K (1978) Driver gas contamination in a high-enthalpy reflected shock tunnel. AIAA J 16(3):277-279

Stalker RJ (1966) The free piston shock tube. Aeronautic Quarter 17:351-370

Stalker RJ (2006) Modern developments in hypersonic wind tunnels. Aeronautic J 110(1103):21-40

Stalker RJ, Paull A, Mee DJ, Morgan RG, Jacobs PA (2005) Scramjets and shock tunnels - The Queensland Experience. Progress Aerospace Sci 41(6):471-513

Stennett SJ, Gildfind D, Jacobs P, Morgan R, James C, Toniato P (2020) The X3R free-piston reflected shock tunnel: Australia's new large-scale, long-duration hypersonic testing capability. In: 23rd AIAA International Space Planes and Hypersonic Systems and Technologies Conference

Stennett SJ, Gildfind DE, Jacobs PA, Morgan RG (2018) Performance optimization of X3R: A new reflected shock tunnel mode for the
X3 expansion tube. In: 2018 Aerodynamic Measurement Technology and Ground Testing Conference

Subiah SD, Collen PL, Doherty LJ, McGilvray M (2019) Condition development and commissioning of the Oxford T6 Stalker Tunnel in Reflected Shock Tunnel Mode. In: International Conference on Flight Vehicles, Aerothermodynamics and Re-entry Missions \& Engineering

Tamagno J, Bakos R, Pulsonetti M, Erdos J (1990) Hypervelocity real gas capabilities of GASL's expansion tube (HYPULSE) facility. In: 16th Aerodynamic Ground Testing Conference, page 1390

Tanno H, Itoh K, Komuro T, Sato K (2000) Experimental study on the tuned operation of a free piston driver. Shock Waves 10(1):1-7

Tanno H, Komuro T, Sato K, Itoh K, Arai K, Yamada K (2016) Basic characteristics of the free-piston driven expansion tube JAXA HEK-X. In: 32nd AIAA Aerodynamic Measurement Technology and Ground Testing Conference

Trimpi RL (1962) A preliminary theoretical study of the expansion tube, a new device for producing high-enthalpy short-duration hypersonic gas flows. Technical Report R-133, NASA

Tsai C-Y, Chue R, Nicholson C, Tyll J (2009) Hypervelocity capability of HyPulse Shock Tunnel for radiative heat transfer measurements at lunar reentries. In: 47th AIAA Aerospace Sciences Meeting

Wagner A (12-13 April 2018) High frequency pressure measurements in shock tunnels using piezoelectric transducers. In: 12th International Workshop on Shock Tube Technology, Kakuda, Japan

Wang Q, Li JW, Lu P, Li JP, Zhao W, Jiang Z (2017) Pressure and heat flux calibration of the long-test-duration hypervelocity detonationdriven shock tunnel. In: 21st AIAA International Space Planes and Hypersonics Technologies Conference, page 2155

White DR (1958) Influence of diaphragm opening time on shock-tube flows. J Fluid Mech 4(6):585-599

Wilcox DC et al (1998) Turbulence modeling for CFD, vol 2. DCW industries, $\mathrm{La}$

Wilke CR (1950) A viscosity equation for gas mixtures. J Chem hys 18(4):517-519

Wittliff CE (1959) The tailored-interface hypersonic shock tunnel. J Aerospace Sci 26(4):219-228

Yamada G, Suzuki T, Takayanagi H, Fujita K (2011) Development of shock tube for ground testing reentry aerothermodynamics. Trans Japan Soc Aeronautic Space Sci 54(183):51-61

Zoby EV, Sullivan EM (1966) Effects of corner radius on stagnationpoint velocity gradients on blunt axisymmetric bodies. J Spacecraft Rockets 3(10):1567-1567

Publisher's Note Springer Nature remains neutral with regard to jurisdictional claims in published maps and institutional affiliations. 